\title{
On Efficient Second Order Stabilized Semi-Implicit Schemes for the Cahn-Hilliard Phase-Field Equation
}

\author{
Lin Wang · Haijun Yu
}

May 23, 2018

\begin{abstract}
Efficient and energy stable high order time marching schemes are very important but not easy to construct for the study of nonlinear phase dynamics. In this paper, we propose and study two linearly stabilized second order semi-implicit schemes for the Cahn-Hilliard phase-field equation. One uses backward differentiation formula and the other uses CrankNicolson method to discretize linear terms. In both schemes, the nonlinear bulk forces are treated explicitly with two second-order stabilization terms. This treatment leads to linear elliptic systems with constant coefficients, for which lots of robust and efficient solvers are available. The discrete energy dissipation properties are proved for both schemes. Rigorous error analysis is carried out to show that, when the time step-size is small enough, second order accuracy in time is obtained with a prefactor controlled by a fixed power of $1 / \varepsilon$, where $\varepsilon$ is the characteristic interface thickness. Numerical results are presented to verify the accuracy and efficiency of proposed schemes.
\end{abstract}

Keywords phase field model · Cahn-Hilliard equation · energy stable $\cdot$ stabilized semi-implicit scheme $\cdot$ second order time marching

Mathematics Subject Classification (2000) $65 \mathrm{M} 12 \cdot 65 \mathrm{M} 15 \cdot 65 \mathrm{P} 40$

\section{Introduction}

In this paper, we consider numerical approximation for the Cahn-Hilliard equation

$$
\begin{cases}\phi_{t}=\gamma \Delta \mu, & (x, t) \in \Omega \times(0, T], \\ \mu=-\varepsilon \Delta \phi+\frac{1}{\varepsilon} f(\phi), & (x, t) \in \Omega \times(0, T], \\ \left.\phi\right|_{t=0}=\phi_{0}(x), & x \in \Omega,\end{cases}
$$

L. Wang · H. Yu (四)

E-mail: wanglin@lsec.cc.ac.cn (L. Wang), E-mail: hyu@lsec.cc.ac.cn (H. Yu)

School of Mathematical Sciences, University of Chinese Academy of Sciences, Beijing 100049, China. NCMIS \& LSEC, Institute of Computational Mathematics and Scientific/Engineering Computing, Academy of Mathematics and Systems Science, Beijing 100190, China. 
with Neumann boundary condition

$$
\partial_{n} \phi=0, \quad \partial_{n} \mu=0, \quad x \in \partial \Omega .
$$

Here $\Omega \in R^{d}, d=2,3$ is a bounded domain with a locally Lipschitz boundary, $n$ is the outward normal, $T$ is a given time, $\phi(x, t)$ is the phase-field variable. Function $f(\phi)=F^{\prime}(\phi)$, and $F(\phi)$ is a given energy potential with two local minima, e.g. the double well potential $F(\phi)=\frac{1}{4}\left(\phi^{2}-1\right)^{2}$. The two minima of $F$ produce two phases, with the typical thickness of the interface between two phases given by $\varepsilon$. $\gamma$, called mobility, is related to the characteristic relaxation time of the system.

The Cahn-Hilliard equation originated from the work by Cahn and Hilliard [6], in which a diffusive model of interfacial energy is built to describe the phase separation and coarsening phenomena in non-uniform systems. If the term $\Delta \mu$ in equation (1.1) is replaced with $-\mu$, one get the Allen-Cahn equation, which was introduced by Allen and Cahn [1] to describe the motion of anti-phase boundaries in crystalline solids. The Cahn-Hilliard equation and the Allen-Cahn equation are two widely used phase-field models. In a phase-field model, the information of interface is implicitly determined by a smooth phase function $\phi$. In most parts of the domain $\Omega$, the value of $\phi$ is close to local minima of $F$. The interface is a thin layer of thickness $\varepsilon$ connecting regions of different local minima. It is easier to deal with dynamical process involving morphology changes of interfaces using phase-field models due to the good mathematical properties that phase-field equations have. For this reason, phase field models have been the subject of many theoretical and numerical investigations for several decades(cf., for instance, [12], [15], [9], [5], [13], [16], [21], [34], [18], [30], [38], [10]).

But, numerically solving the phase-field equations is not an easy task, especially for the Cahn-Hilliard equation. Firstly, the small parameter $\varepsilon$ requires very high spatial and temporal grid resolutions. Secondly, the small parameter $\varepsilon$ in the nonlinear bulk force $f(\phi)$ and the bi-harmonic operator makes the equation very stiff, which make it very hard to solve. Nevertheless, lots of numerical schemes have been proposed to solve the Cahn-Hilliard equation based on its mathematical properties. Two most important properties of the Cahn-Hilliard equation are the volume conservation property

$$
\int_{\Omega} \phi(x, t) d x=\int_{\Omega} \phi_{0}(x) d x, \quad \forall t>0
$$

and energy dissipation property

$$
E_{\varepsilon}(\phi(\cdot, t))-E_{\varepsilon}\left(\phi_{0}\right)=-\gamma \int_{0}^{t} \int_{\Omega}|\nabla \mu|^{2} d x=-\gamma \int_{0}^{t}\left\|\phi_{t}\right\|_{-1}^{2} d x, \quad \forall t>0,
$$

where $E_{\varepsilon}$ is the free energy functional defined as

$$
E_{\varepsilon}(\phi):=\int_{\Omega}\left(\frac{\varepsilon}{2}|\nabla \phi|^{2}+\frac{1}{\varepsilon} F(\phi)\right) d x .
$$

Here the $H^{-1}$ norm $\|\cdot\|_{-1}$ is defined in Section 2. Since the nonlinear bulk energy $F$ is neither a convex nor a concave function, treating it fully explicit or implicit in a time discretization will not lead to an efficient scheme. In fact, if the nonlinear force $f$ is treated fully explicitly, the resulting scheme will require a very tiny step-size to be stable (cf. for instance [38]). On the other hand, treating it fully implicitly will lead to a nonlinear system, for which the solution existence and uniqueness requires a restriction on step-size as well (cf. e.g. [18]). One popular approach to solve this dilemma is the convex splitting method, which appears to be introduced by Elliott and Stuart [14] and popularized by Eyre [16]. In a convex splitting 
approach, the convex part of $F$ is treated implicitly and the concave part treated explicitly. The convex splitting scheme given in [14][16] is of first order accurate and unconditional stable. In each time step, one need solve a nonlinear system. The solution existence and uniqueness is guaranteed since the nonlinear system corresponds to a convex optimization problem. The convex splitting method was used widely, and several second order extensions were derived in different situations $[10,3,8,25]$. Another type of energy stable schemes is the secant-line method proposed by $\mathrm{Du}$ and Nicolaides [12]. It is also used and extended in several other works, e.g. [21,31,17,10,22,3,50,4]. Like the fully implicit method, the usual second order convex splitting method and the secant-type method for Cahn-Hilliard equation need a small time step-size to guarantee the semi-discretized nonlinear system has a unique solution (cf. for instance $[12,2])$. To remove the restriction on time step-size, diffusive three-step CrankNicolson schemes coupled with a second order convex splitting were introduced in [25] and [11], In which, the time semi-discretized system is a nonlinear but unique solvable problem.

Recently, a new approach termed as invariant energy quadratization (IEQ) was introduced to handle the nonlinear energy. When applying to Cahn-Hilliard equation, it first appeared in $[23,24]$ as a Lagrange multiplier method. It then generalized by Yang et al. and successfully extended to handle several very complicated nonlinear phase-field models [46,27,47,48]. In the IEQ approach, a new variable which equals to the square root of $F$ is introduced, so the energy is written into a quadratic form in terms of the new variable. By using semiimplicit treatments to all the nonlinear terms in the equations, one get a linear and energy stable scheme. It is straightforward to prove the unconditional stability for both first order and second order IEQ schemes. Comparing to the convex splitting approach, IEQ leads to well-structured linear system which is easier to solve. The modified energy in IEQ is an orderconsistent approximation to the original system energy. At each time step, it needs to solve a linear system with time-varying coefficients. To avoid the variable-coefficient system, a new approach called scalar auxiliary variable (SAV) was introduced by Shen et al.[36,37] recently. The methodology of SAV is very similar to IEQ, but at each time step, only systems with constant coefficients need to be solved.

Another trend of improving numerical schemes for phase-field models focuses on algorithm efficiency. Chen and Shen [7] and Zhu et al. [51] studied stabilized semi-implicit Fourier-spectral method for Cahn-Hilliard equation. The space variables are discretized using a Fourier-spectral method whose convergence rate is exponential in contrast to the second order of a usual finitedifference method. The time variable is discretized by using semi-implicit schemes which allow much larger time step sizes than explicit schemes. Xu and Tang [44] introduced a different stabilized term to build large time-stepping stabilized semi-implicit method for a 2-dimensional epitaxial growth model. He et al [28] proposed a similar large time-stepping methods for the Cahn-Hilliard equation, in which a stabilized term $B\left(\phi^{n+1}-\phi^{n}\right)\left(\right.$ resp. $\left.B\left(\phi^{n+1}-2 \phi^{n}+\phi^{n-1}\right)\right)$ is added to the nonlinear bulk force for the first order(resp. second order) scheme. Shen and Yang applied similar stabilization skill to Allen-Cahn equation and Cahn-Hilliard equation in mixed formulation [38], which leads to unconditionally energy stable first-order linear schemes and second-order linear schemes with reasonable stability conditions. This idea was followed up in [20] for the stabilized Crank-Nicolson schemes for phase field models. Another stabilized second-order Crank-Nicolson scheme with a new convex-concave splitting of the energy is proposed for a tumor-growth system by $\mathrm{Wu}$ et al.[43]. Those time marching schemes all lead to linear systems, which are easier to solve than nonlinear systems resulting from traditional convex-splitting schemes, in which the nonlinear convex force is treated implicitly. On the other hand, when the nonlinear force is treated explicitly, one need to introduce a proper stabilization term and a suitably truncated nonlinear function $\tilde{f}(\phi)$ instead of $f(\phi)$ to prove the unconditionally energy stable property with a reasonable stabilization constant. It is worth 
to mention that with no truncation made to $f(\phi)$, Li et al $[33,32]$ proved that the energy stable property can be obtained as well, but a much larger stability constant need be used. The stabilization skill has also been used in constructing higher order schemes, for example the exponential time differencing (ETD) scheme [29] and Runge-Kutta scheme [26,40].

In this paper, we study the stability and convergence properties of two new second-order semi-implicit time marching schemes. One uses second-order backward differentiation formula (BDF2) and other one uses Crank-Nicolson approximation. In both schemes, explicit extrapolation are used for the nonlinear force with two order-consistent extra stabilization terms added to guarantee the energy dissipation. We also give an optimal error analysis in $l^{\infty}\left(0, T ; H^{-1}\right) \cap l^{2}\left(0, T ; H^{1}\right)$ norm. The new methods have several merits: 1$)$ They are second order accurate; 2 ) They lead to linear systems with constant coefficients after time discretization; 3) Discrete energy dissipations are proved. The proofs base on Galerkin formulation. Both finite element method and spectral method can be used for spatial discretization to conserve volume fraction and satisfy discretized energy dissipation law.

The remain part of this paper is organized as follows. In Section 2, we present the two second-order stabilized schemes for the Cahn-Hilliard equation and prove they are energy stable. In Section 3, we present an error estimate for the BDF2 scheme to derive a convergence rate that does not depend on $1 / \varepsilon$ exponentially. Implementation details and numerical results for a test problem in a 2-dimensional square domain are presented in Section 4 to verify our theoretical results. We end the paper with some concluding remarks in Section 5.

\section{The two second order stabilized linear schemes}

We first introduce some notations which will be used throughout the paper. We use $\|\cdot\|_{m, p}$ to denote the standard norm of the Sobolev space $W^{m, p}(\Omega)$. In particular, we use $\|\cdot\|_{L^{p}}$ to denote the norm of $W^{0, p}(\Omega)=L^{p}(\Omega) ;\|\cdot\|_{m}$ to denote the norm of $W^{m, 2}(\Omega)=H^{m}(\Omega)$; and $\|\cdot\|$ to denote the norm of $W^{0,2}(\Omega)=L^{2}(\Omega)$. Let $(\cdot, \cdot)$ represent the $L^{2}$ inner product. In addition, define for $p \geq 0$

$$
H^{-p}(\Omega):=\left(H^{p}(\Omega)\right)^{*}, \quad H_{0}^{-p}(\Omega):=\left\{u \in H^{-p}(\Omega) \mid\langle u, 1\rangle_{p}=0\right\}
$$

where $\langle\cdot, \cdot\rangle_{p}$ stands for the dual product between $H^{p}(\Omega)$ and $H^{-p}(\Omega)$. We denote $L_{0}^{2}(\Omega):=$ $H_{0}^{0}(\Omega)$. For $v \in L_{0}^{2}(\Omega)$, let $-\Delta^{-1} v:=v_{1} \in H^{1}(\Omega) \cap L_{0}^{2}(\Omega)$, where $v_{1}$ is the solution to

$$
-\Delta v_{1}=v \text { in } \Omega, \quad \frac{\partial v_{1}}{\partial n}=0 \text { on } \partial \Omega,
$$

and $\|v\|_{-1}:=\sqrt{\left(v,-\Delta^{-1} v\right)}$.

For any given function $\phi(t)$ of $t$, we use $\phi^{n}$ to denote an approximation of $\phi(n \tau)$, where $\tau$ is the step-size. We will frequently use the shorthand notations: $\delta_{t} \phi^{n+1}:=\phi^{n+1}-\phi^{n}, \delta_{t t} \phi^{n+1}:=$ $\phi^{n+1}-2 \phi^{n}+\phi^{n-1}, D_{\tau} \phi^{n+1}:=\frac{3 \phi^{n+1}-4 \phi^{n}+\phi^{n-1}}{2 \tau}=\frac{1}{\tau} \delta_{t} \phi^{n+1}+\frac{1}{2 \tau} \delta_{t t} \phi^{n+1}, \hat{\phi}^{n+\frac{1}{2}}:=\frac{3}{2} \phi^{n}-\frac{1}{2} \phi^{n-1}$ and $\hat{\phi}^{n+1}:=2 \phi^{n}-\phi^{n-1}$. Following identities will be used frequently as well

$$
\begin{gathered}
2\left(h^{n+1}-h^{n}, h^{n+1}\right)=\left\|h^{n+1}\right\|^{2}-\left\|h^{n}\right\|^{2}+\left\|h^{n+1}-h^{n}\right\|^{2}, \\
\left(D_{\tau} h^{n+1}, h^{n+1}\right)=\frac{1}{4 \tau}\left(\left\|h^{n+1}\right\|^{2}+\left\|2 h^{n+1}-h^{n}\right\|^{2}-\left\|h^{n}\right\|^{2}-\left\|2 h^{n}-h^{n-1}\right\|^{2}+\left\|\delta_{t t} h^{n+1}\right\|^{2}\right) .
\end{gathered}
$$


To prove energy stability of the numerical schemes, we assume that the derivative of $f$ in equation (1.1) is uniformly bounded, i.e.

$$
\max _{\phi \in \mathbf{R}}\left|f^{\prime}(\phi)\right| \leq L,
$$

where $L$ is a non-negative constant.

Note that, if the phase-field system satisfies the maximum principle, then (2.3) is satisfied for any smooth $f$. Although the Cahn-Hilliard equation does not satisfy the maximum principle, it has been shown that in [5] that for a truncated potential $F$ with quadratic growth at infinities, the maximum norm of the solution to the Cahn-Hilliard equation is bounded. On the other hand, for a more general potential F, Feng and Prohl [19] proved that if the Cahn-Hilliard equation convergence to its sharp-interface limit, then its solution has a $L^{\infty}$ bound. Therefore, it has been a common practice (cf. $[30,38,10])$ to consider the Cahn-Hilliard equations with a truncated double-well potential $F$ such that $(2.3)$ is satisfied.

2.1 The stabilized linear Crank-Nicolson scheme

Suppose $\phi^{0}=\phi_{0}(\cdot)$ and $\phi^{1} \approx \phi(\cdot, \tau)$ are given, our stabilized liner Crank-Nicolson scheme (abbr. SL-CN) calculates $\phi^{n+1}, n=1,2, \ldots, N=T / \tau-1$ iteratively, using

$$
\begin{gathered}
\frac{\phi^{n+1}-\phi^{n}}{\tau}=\gamma \Delta \mu^{n+\frac{1}{2}}, \\
\mu^{n+\frac{1}{2}}=-\varepsilon \Delta\left(\frac{\phi^{n+1}+\phi^{n}}{2}\right)+\frac{1}{\varepsilon} f\left(\frac{3}{2} \phi^{n}-\frac{1}{2} \phi^{n-1}\right)-A \tau \Delta \delta_{t} \phi^{n+1}+B \delta_{t t} \phi^{n+1},
\end{gathered}
$$

where $A$ and $B$ are two non-negative constants to stabilize the scheme.

Theorem 1 Assume (2.3) is satisfied. Under the condition

$$
A \geq \frac{L^{2}}{16 \varepsilon^{2}} \gamma, \quad B \geq \frac{L}{2 \varepsilon}
$$

the following energy dissipation law

$$
E_{C}^{n+1} \leq E_{C}^{n}-\left(2 \sqrt{\frac{A}{\gamma}}-\frac{L}{2 \varepsilon}\right)\left\|\delta_{t} \phi^{n+1}\right\|^{2}-\left(\frac{B}{2}-\frac{L}{4 \varepsilon}\right)\left\|\delta_{t t} \phi^{n+1}\right\|^{2}, \quad \forall n \geq 1,
$$

holds for the scheme (2.4)-(2.5), where

$$
E_{C}^{n+1}=E_{\varepsilon}\left(\phi^{n+1}\right)+\left(\frac{L}{4 \varepsilon}+\frac{B}{2}\right)\left\|\delta_{t} \phi^{n+1}\right\|^{2}
$$

Proof Pairing (2.4) with $\tau \mu^{n+\frac{1}{2}},(2.5)$ with $-\delta_{t} \phi^{n+1}$, and combining the results, we get

$$
\begin{aligned}
& \frac{\varepsilon}{2}\left(\left\|\nabla \phi^{n+1}\right\|^{2}-\left\|\nabla \phi^{n}\right\|^{2}\right)+\frac{1}{\varepsilon}\left(f\left(\hat{\phi}^{n+\frac{1}{2}}\right), \delta_{t} \phi^{n+1}\right) \\
= & -\gamma \tau\left\|\nabla \mu^{n+\frac{1}{2}}\right\|^{2}-A \tau\left\|\nabla \delta_{t} \phi^{n+1}\right\|^{2}-B\left(\delta_{t t} \phi^{n+1}, \delta_{t} \phi^{n+1}\right) .
\end{aligned}
$$

Pairing (2.4) with $2 \sqrt{\frac{A}{\gamma}} \tau \delta_{t} \phi^{n+1}$, then using Cauchy-Schwartz inequality, we get

$$
2 \sqrt{\frac{A}{\gamma}}\left\|\delta_{t} \phi^{n+1}\right\|^{2}=-2 \sqrt{A \gamma} \tau\left(\nabla \mu^{n+\frac{1}{2}}, \nabla \delta_{t} \phi^{n+1}\right) \leq \gamma \tau\left\|\nabla \mu^{n+\frac{1}{2}}\right\|^{2}+A \tau\left\|\nabla \delta_{t} \phi^{n+1}\right\|^{2} .
$$


To handle the term involving $f$, we expand $F\left(\phi^{n+1}\right)$ and $F\left(\phi^{n}\right)$ at $\hat{\phi}^{n+\frac{1}{2}}$ as

$$
\begin{gathered}
F\left(\phi^{n+1}\right)=F\left(\hat{\phi}^{n+\frac{1}{2}}\right)+f\left(\hat{\phi}^{n+\frac{1}{2}}\right)\left(\phi^{n+1}-\hat{\phi}^{n+\frac{1}{2}}\right)+\frac{1}{2} f^{\prime}\left(\xi_{1}^{n}\right)\left(\phi^{n+1}-\hat{\phi}^{n+\frac{1}{2}}\right)^{2}, \\
F\left(\phi^{n}\right)=F\left(\hat{\phi}^{n+\frac{1}{2}}\right)+f\left(\hat{\phi}^{n+\frac{1}{2}}\right)\left(\phi^{n}-\hat{\phi}^{n+\frac{1}{2}}\right)+\frac{1}{2} f^{\prime}\left(\xi_{2}^{n}\right)\left(\phi^{n}-\hat{\phi}^{n+\frac{1}{2}}\right)^{2},
\end{gathered}
$$

where $\xi_{1}^{n}$ is a number between $\phi^{n+1}$ and $\hat{\phi}^{n+\frac{1}{2}}, \xi_{2}^{n}$ is a number between $\phi^{n}$ and $\hat{\phi}^{n+\frac{1}{2}}$. Taking the difference of above two equations, we have

$$
\begin{aligned}
& F\left(\phi^{n+1}\right)-F\left(\phi^{n}\right)-f\left(\hat{\phi}^{n+\frac{1}{2}}\right)\left(\phi^{n+1}-\phi^{n}\right) \\
= & \frac{1}{2} f^{\prime}\left(\xi_{1}^{n}\right)\left[\left(\phi^{n+1}-\hat{\phi}^{n+\frac{1}{2}}\right)^{2}-\left(\phi^{n}-\hat{\phi}^{n+\frac{1}{2}}\right)^{2}\right]-\frac{1}{2}\left(f^{\prime}\left(\xi_{2}^{n}\right)-f^{\prime}\left(\xi_{1}^{n}\right)\right)\left(\phi^{n}-\hat{\phi}^{n+\frac{1}{2}}\right)^{2} \\
= & \frac{1}{2} f^{\prime}\left(\xi_{1}^{n}\right) \delta_{t} \phi^{n+1} \delta_{t t} \phi^{n+1}-\frac{1}{8}\left(f^{\prime}\left(\xi_{2}^{n}\right)-f^{\prime}\left(\xi_{1}^{n}\right)\right)\left(\delta_{t} \phi^{n}\right)^{2} \\
\leq & \frac{L}{4}\left(\left|\delta_{t} \phi^{n+1}\right|^{2}+\left|\delta_{t t} \phi^{n+1}\right|^{2}\right)+\frac{L}{4}\left|\delta_{t} \phi^{n}\right|^{2} .
\end{aligned}
$$

Multiplying the above equation with $\frac{1}{\varepsilon}$, then taking integration leads to

$$
\frac{1}{\varepsilon}\left(F\left(\phi^{n+1}\right)-F\left(\phi^{n}\right)-f\left(\hat{\phi}^{n+\frac{1}{2}}\right) \delta_{t} \phi^{n+1}, 1\right) \leq \frac{L}{4 \varepsilon}\left(\left\|\delta_{t} \phi^{n+1}\right\|^{2}+\left\|\delta_{t t} \phi^{n+1}\right\|^{2}+\left\|\delta_{t} \phi^{n}\right\|^{2}\right) .
$$

For the term involving $B$, by using identity (2.1) with $h^{n+1}=\delta_{t} \phi^{n+1}$, one get

$$
-B\left(\delta_{t t} \phi^{n+1}, \delta_{t} \phi^{n+1}\right)=-\frac{B}{2}\left\|\delta_{t} \phi^{n+1}\right\|^{2}+\frac{B}{2}\left\|\delta_{t} \phi^{n}\right\|^{2}-\frac{B}{2}\left\|\delta_{t t} \phi^{n+1}\right\|^{2}
$$

Summing up (2.9)-(2.12), we obtain

$$
\begin{aligned}
& \frac{\varepsilon}{2}\left(\left\|\nabla \phi^{n+1}\right\|^{2}-\left\|\nabla \phi^{n}\right\|^{2}\right)+\frac{1}{\varepsilon}\left(F\left(\phi^{n+1}\right)-F\left(\phi^{n}\right), 1\right)+\frac{B}{2}\left\|\delta_{t} \phi^{n+1}\right\|^{2}-\frac{B}{2}\left\|\delta_{t} \phi^{n}\right\|^{2} \\
\leq & -2 \sqrt{\frac{A}{\gamma}}\left\|\delta_{t} \phi^{n+1}\right\|^{2}+\frac{L}{4 \varepsilon}\left\|\delta_{t} \phi^{n+1}\right\|^{2}+\frac{L}{4 \varepsilon}\left\|\delta_{t} \phi^{n}\right\|^{2}-\frac{B}{2}\left\|\delta_{t t} \phi^{n+1}\right\|^{2}+\frac{L}{4 \varepsilon}\left\|\delta_{t t} \phi^{n+1}\right\|^{2},
\end{aligned}
$$

which is the energy estimate (2.7).

\subsection{The stabilized linear BDF2 scheme}

Suppose $\phi^{0}=\phi_{0}(\cdot)$ and $\phi^{1} \approx \phi(\cdot, \tau)$ are given, our stabilized linear BDF2 scheme (abbr. SL-BDF2) calculate $\phi^{n+1}, n=1,2, \ldots, N=T / \tau-1$ iteratively, using

$$
\begin{gathered}
\frac{3 \phi^{n+1}-4 \phi^{n}+\phi^{n-1}}{2 \tau}=\gamma \Delta \mu^{n+1}, \\
\mu^{n+1}=-\varepsilon \Delta \phi^{n+1}+\frac{1}{\varepsilon} f\left(2 \phi^{n}-\phi^{n-1}\right)-A \tau \Delta \delta_{t} \phi^{n+1}+B \delta_{t t} \phi^{n+1},
\end{gathered}
$$

where $A$ and $B$ are two non-negative constants. 
Theorem 2 Assume (2.3) is satisfied, and

$$
\frac{1}{|\Omega|} \int_{\Omega} \phi^{1} d x=\frac{1}{|\Omega|} \int_{\Omega} \phi^{0} d x=m_{0}
$$

Then under the condition

$$
B \geq \frac{L}{\varepsilon} ; \quad A \geq \frac{\gamma}{\alpha_{2}} \frac{L^{2}}{16 \varepsilon^{2}}-\alpha_{1} \frac{\varepsilon}{2 \tau}, \quad 0 \leq \alpha_{1} \leq 1, \quad 0<\alpha_{2} \leq 1,
$$

the following energy dissipation law

$$
\begin{aligned}
E_{B}^{n+1} \leq E_{B}^{n} & -\frac{1}{4 \tau \gamma}\left\|\delta_{t t} \phi^{n+1}\right\|_{-1}^{2}-\left(1-\alpha_{1}\right) \frac{\varepsilon}{2}\left\|\nabla \delta_{t} \phi^{n+1}\right\|^{2}-\left(1-\alpha_{2}\right) \frac{1}{\tau \gamma}\left\|\delta_{t} \phi^{n+1}\right\|_{-1}^{2} \\
& -\left(2 \sqrt{\frac{\alpha_{2}}{\gamma}\left(A+\frac{\alpha_{1} \varepsilon}{2 \tau}\right)}-\frac{L}{2 \varepsilon}\right)\left\|\delta_{t} \phi^{n+1}\right\|^{2}-\left(\frac{B}{2}-\frac{L}{2 \varepsilon}\right)\left\|\delta_{t t} \phi^{n+1}\right\|^{2}, \quad \forall n \geq 1,
\end{aligned}
$$

holds for the scheme (2.14)-(2.15), where

$$
E_{B}^{n+1}=E_{\varepsilon}\left(\phi^{n+1}\right)+\frac{1}{4 \tau \gamma}\left\|\delta_{t} \phi^{n+1}\right\|_{-1}^{2}+\left(\frac{L}{2 \varepsilon}+\frac{B}{2}\right)\left\|\delta_{t} \phi^{n+1}\right\|^{2}
$$

Proof 1) Integration both sides of equation (2.14), then using the Neumann boundary condition of $\mu$ and property (2.16), we get

$$
\frac{1}{|\Omega|} \int_{\Omega} \phi^{n+1} d x=m_{0}, \quad n=1, \ldots, N
$$

Thus $\delta_{t} \phi^{n+1} \in L_{0}^{2}$ for $n=0, \ldots, N$.

2) Pairing (2.14) with $(-\Delta)^{-1} \delta_{t} \phi^{n+1} / \gamma$, plus (2.15) paired with $-\delta_{t} \phi^{n+1}$, we get

$$
\begin{aligned}
\left(\frac{1}{\gamma} D_{\tau} \phi^{n+1},(-\Delta)^{-1} \delta_{t} \phi^{n+1}\right)= & \varepsilon\left(\Delta \phi^{n+1}, \delta_{t} \phi^{n+1}\right)-\frac{1}{\varepsilon}\left(f\left(\hat{\phi}^{n+1}\right), \delta_{t} \phi^{n+1}\right) \\
& -A \tau\left\|\nabla \delta_{t} \phi^{n+1}\right\|^{2}-B\left(\delta_{t t} \phi^{n+1}, \delta_{t} \phi^{n+1}\right) .
\end{aligned}
$$

By integration by parts and applying (2.1), (2.2), following identities hold

$$
\begin{aligned}
& -\left(\frac{1}{\gamma} D_{\tau} \phi^{n+1},(-\Delta)^{-1} \delta_{t} \phi^{n+1}\right) \\
= & -\frac{1}{\tau \gamma}\left\|\delta_{t} \phi^{n+1}\right\|_{-1}^{2}-\frac{1}{4 \tau \gamma}\left(\left\|\delta_{t} \phi^{n+1}\right\|_{-1}^{2}-\left\|\delta_{t} \phi^{n}\right\|_{-1}^{2}+\left\|\delta_{t t} \phi^{n+1}\right\|_{-1}^{2}\right), \\
& \varepsilon\left(\Delta \phi^{n+1}, \delta_{t} \phi^{n+1}\right)=-\frac{\varepsilon}{2}\left(\left\|\nabla \phi^{n+1}\right\|^{2}-\left\|\nabla \phi^{n}\right\|^{2}+\left\|\nabla \delta_{t} \phi^{n+1}\right\|^{2}\right), \\
- & B\left(\delta_{t t} \phi^{n+1}, \delta_{t} \phi^{n+1}\right)=-\frac{B}{2}\left\|\delta_{t} \phi^{n+1}\right\|^{2}+\frac{B}{2}\left\|\delta_{t} \phi^{n}\right\|^{2}-\frac{B}{2}\left\|\delta_{t t} \phi^{n+1}\right\|^{2} .
\end{aligned}
$$

To handle the term involves $f$ in $(2.21)$, we expand $F\left(\phi^{n+1}\right)$ and $F\left(\phi^{n}\right)$ at $\hat{\phi}^{n+1}$ as

$$
\begin{aligned}
F\left(\phi^{n+1}\right) & =F\left(\hat{\phi}^{n+1}\right)+f\left(\hat{\phi}^{n+1}\right)\left(\phi^{n+1}-\hat{\phi}^{n+1}\right)+\frac{1}{2} f^{\prime}\left(\zeta_{1}^{n}\right)\left(\phi^{n+1}-\hat{\phi}^{n+1}\right)^{2}, \\
F\left(\phi^{n}\right) & =F\left(\hat{\phi}^{n+1}\right)+f\left(\hat{\phi}^{n+1}\right)\left(\phi^{n}-\hat{\phi}^{n+1}\right)+\frac{1}{2} f^{\prime}\left(\zeta_{2}^{n}\right)\left(\phi^{n}-\hat{\phi}^{n+1}\right)^{2},
\end{aligned}
$$


where $\zeta_{1}^{n}$ is a number between $\phi^{n+1}$ and $\hat{\phi}^{n+1}, \zeta_{2}^{n}$ is a number between $\phi^{n}$ and $\hat{\phi}^{n+1}$. Taking the difference of above two equations, using the fact $\phi^{n+1}-\hat{\phi}^{n+1}=\delta_{t t} \phi^{n+1}$ and $\phi^{n}-\hat{\phi}^{n+1}=-\delta_{t} \phi^{n}$, we obtain

$$
\begin{aligned}
F\left(\phi^{n+1}\right)-F\left(\phi^{n}\right)-f\left(\hat{\phi}^{n+1}\right) \delta_{t} \phi^{n+1} & =\frac{1}{2} f^{\prime}\left(\zeta_{1}^{n}\right)\left(\delta_{t t} \phi^{n+1}\right)^{2}-\frac{1}{2} f^{\prime}\left(\zeta_{2}^{n}\right)\left(\delta_{t} \phi^{n}\right)^{2} \\
& \leq \frac{L}{2}\left|\delta_{t t} \phi^{n+1}\right|^{2}+\frac{L}{2}\left|\delta_{t} \phi^{n}\right|^{2}
\end{aligned}
$$

Taking inner product of the above equation with constant $1 / \varepsilon$, then combining the result with (2.21), (2.22), (2.23) and (2.24), we obtain

$$
\begin{aligned}
& \frac{1}{\varepsilon}\left(F\left(\phi^{n+1}\right)-F\left(\phi^{n}\right), 1\right)+\frac{\varepsilon}{2}\left(\left\|\nabla \phi^{n+1}\right\|^{2}-\left\|\nabla \phi^{n}\right\|^{2}\right) \\
& +\frac{1}{4 \tau \gamma}\left(\left\|\delta_{t} \phi^{n+1}\right\|_{-1}^{2}-\left\|\delta_{t} \phi^{n}\right\|_{-1}^{2}\right)+\left(\frac{L}{2 \varepsilon}+\frac{B}{2}\right)\left(\left\|\delta_{t} \phi^{n+1}\right\|^{2}-\left\|\delta_{t} \phi^{n}\right\|^{2}\right) \\
\leq & -\frac{1}{4 \tau \gamma}\left\|\delta_{t t} \phi^{n+1}\right\|_{-1}^{2}-\frac{1}{\tau \gamma}\left\|\delta_{t} \phi^{n+1}\right\|_{-1}^{2}-\frac{\varepsilon}{2}\left\|\nabla \delta_{t} \phi^{n+1}\right\|^{2}-A \tau\left\|\nabla \delta_{t} \phi^{n+1}\right\|^{2} \\
& +\frac{L}{2 \varepsilon}\left\|\delta_{t} \phi^{n+1}\right\|^{2}-\frac{B}{2}\left\|\delta_{t t} \phi^{n+1}\right\|^{2}+\frac{L}{2 \varepsilon}\left\|\delta_{t t} \phi^{n+1}\right\|^{2} .
\end{aligned}
$$

Combining the above equation and the inequality

$$
\chi\left\|\nabla \delta_{t} \phi^{n+1}\right\|^{2}+\frac{\alpha_{2}}{\tau \gamma}\left\|\delta_{t} \phi^{n+1}\right\|_{-1}^{2} \geq 2 \sqrt{\frac{\chi \alpha_{2}}{\tau \gamma}}\left\|\delta_{t} \phi^{n+1}\right\|^{2},
$$

with $\chi=A \tau+\frac{\alpha_{1} \varepsilon}{2}, 0 \leq \alpha_{1} \leq 1,0<\alpha_{2} \leq 1$, we get the energy estimate (2.18).

Remark 1 The discrete Energy $E_{B}$ defined in equation (2.19) is a second order approximations to the original energy $E$, since $\left\|\delta_{t} \phi^{n+1}\right\|^{2},\left\|\delta_{t} \phi^{n+1}\right\|_{-1}^{2} \sim O\left(\tau^{2}\right)$ provided that the schemes converge. On the other side, summing up the equation (2.18) with $\alpha_{1}=\alpha_{2}=1$ for $n=1, \ldots, N$, we get

$$
E_{B}^{N+1}+\sum_{n=1}^{N}\left(\frac{1}{4 \tau \gamma}\left\|\delta_{t t} \phi^{n+1}\right\|_{-1}^{2}+\beta_{1}\left\|\delta_{t} \phi^{n+1}\right\|^{2}+\beta_{2}\left\|\delta_{t t} \phi^{n+1}\right\|^{2}\right) \leq E_{B}^{1}
$$

where $\beta_{1}=2 \sqrt{\frac{A}{\gamma}+\frac{\varepsilon}{2 \tau \gamma}}-\frac{L}{2 \varepsilon}, \beta_{2}=\frac{B}{2}-\frac{L}{2 \varepsilon}$. By taking $N \rightarrow \infty$, we get $\delta_{t} \phi^{N+1} \rightarrow 0$ and $\delta_{t t} \phi^{N+1} \rightarrow 0$ if $\beta_{1}>0$ and $\beta_{2} \geq 0$, which means the system will eventually converge to a steady state. By equation (2.14) and (2.15), this steady state is an extreme point of the original energy functional $E$. Same argument applies to the LS-CN scheme and similar second order stabilization schemes for the Allen-Cahn equation [42].

Remark 2 The constant $A$ defined in equation (2.6) and (2.17) seems to be quite large when $\varepsilon$ is small, but it is not necessarily true. Since usually $\gamma$ is a small constant related to $\varepsilon$. For example, it was showed in [35] that, the Cahn-Hilliard equation coupled with the Navier-Stokes equations have a sharp-interface limit when $O\left(\varepsilon^{3}\right) \leq \gamma \leq O(\varepsilon)$, while $\gamma \sim O\left(\varepsilon^{2}\right)$ gives the fastest convergence. A similar result is obtained for the Cahn-Hilliard Navier-Stokes system with a more general boundary condition [45]. On the other hand, the numerical results in Section 4 shows $A$ can take much smaller values than those defined in (2.6) and (2.17) when nonzero $B$ values are used. 
Remark 3 From equation (2.17), we see that the SL-BDF2 scheme is stable (equation (2.18) holds with $\alpha_{1}=\alpha_{2}=1$ ) with any $A \geq 0$, if

$$
\tau \leq \frac{8 \varepsilon^{3}}{L^{2} \gamma}
$$

If $\tau$ small enough, the combination of the term $\frac{1}{\tau}\left\|\delta_{t} \phi^{n+1}\right\|_{-1}^{2}$ and term $\frac{\varepsilon}{2}\left\|\nabla \delta_{t} \phi^{n+1}\right\|^{2}$ controls the term $\left\|\delta_{t t} \phi^{n+1}\right\|^{2}$ as well, since

$$
\left\|\delta_{t t} \phi^{n+1}\right\|^{2} \leq 2\left\|\delta_{t} \phi^{n+1}\right\|^{2}+2\left\|\delta_{t} \phi^{n}\right\|^{2} .
$$

A direct calculation shows that the SL-BDF2 scheme is stable with any $A \geq 0, B \geq 0$, if

$$
\tau \leq \frac{8 \varepsilon^{3}}{25 L^{2} \gamma}
$$

\section{Convergence analysis}

In this section, we shall establish the error estimate of the semi-discretized scheme SL-BDF2 for the Cahn-Hilliard equation in the norm of $l^{\infty}\left(0, T ; H^{-1}\right) \cap l^{2}\left(0, T ; H^{1}\right)$. We will shown that, if the interface is well developed in the initial condition, the error bounds depend on $\frac{1}{\varepsilon}$ only in some lower polynomial order for small $\varepsilon$. Similar error estimate result can be obtained for the SL-CN scheme but the analysis is more involved, we put it into [41].

Let $\phi\left(t^{n}\right)$ be the exact solution at time $t=t^{n}$ to equation (1.1) and $\phi^{n}$ be the solution at time $t=t^{n}$ to the time discrete numerical scheme (2.14)-(2.15), we define error function $e^{n}:=\phi^{n}-\phi\left(t^{n}\right)$. Obviously $e^{0}=0$.

Before presenting the detailed error analysis, we first make some assumptions. For simplicity, we take $\gamma=1$ in this section, and assume $0<\varepsilon \ll 1$. We use notation $\lesssim$ in the sense that $f \lesssim g$ means that $f \leq C g$ with a positive constant $C$ independent of $\tau, \varepsilon$.

Assumption 1 We assume that $f$ either satisfies the following properties (i) and (ii), or (i) and (iii).

(i) $F \in C^{4}(\mathbf{R}), F( \pm 1)=0$, and $F>0$ elsewhere. There exist two non-negative constants $B_{0}, B_{1}$, such that

$$
\phi^{2} \leq B_{0}+B_{1} F(\phi), \quad \forall \phi \in \mathbf{R} .
$$

(ii) $f=F^{\prime} . f^{\prime}$ and $f^{\prime \prime}$ are uniformly bounded, or, $f$ satisfies (2.3) and

$$
\max _{\phi \in \mathbf{R}}\left|f^{\prime \prime}(\phi)\right| \leq L_{2}
$$

where $L_{2}$ is a non-negative constant.

(iii) $f$ satisfies for some finite $2 \leq p \leq 3+\frac{d}{3(d-2)}$ and positive numbers $\tilde{c}_{i}>0, i=0, \ldots, 5$,

$$
\begin{gathered}
\tilde{c}_{1}|\phi|^{p-2}-\tilde{c}_{0} \leq f^{\prime}(\phi) \leq \tilde{c}_{2}|\phi|^{p-2}+\tilde{c}_{3}, \\
\left|f^{\prime \prime}(\phi)\right| \leq \tilde{c}_{4}|\phi|^{(p-3)^{+}}+\tilde{c}_{5},
\end{gathered}
$$

where for any real number $a$, the notation $(a)^{+}:=\max \{a, 0\}$. 
Note that Assumption 1 (ii) is a special case of Assumption 1 (iii) with $p=2$. The commonly-used quartic double-well potential satisfies Assumption (i) and (iii) with $p=4$. Furthermore, from equation (3.3) we easily get

$$
-\left(f^{\prime}(\phi) u, u\right) \leq \tilde{c}_{0}\|u\|^{2}, \quad \forall u \in L^{2}(\Omega) .
$$

Assumption 2 We assume that $\phi^{0}$ is smooth enough. More precisely, there exist constant $m_{0}$ and non-negative constants $\sigma_{1}, \ldots, \sigma_{6}$, such that

$$
\begin{gathered}
m_{0}:=\frac{1}{|\Omega|} \int_{\Omega} \phi^{0}(x) \mathrm{d} x \in(-1,1), \\
E_{\varepsilon}\left(\phi^{0}\right):=\frac{\varepsilon}{2}\left\|\nabla \phi^{0}\right\|^{2}+\frac{1}{\varepsilon}\left\|F\left(\phi^{0}\right)\right\|_{L^{1}} \lesssim \varepsilon^{-\sigma_{1}}, \\
\left\|\phi_{t}^{0}\right\|_{-1}^{2} \lesssim \varepsilon^{-\sigma_{2}}, \\
\left\|\phi_{t}^{0}\right\|^{2} \lesssim \varepsilon^{-\sigma_{3}} ; \\
\varepsilon\left\|\nabla \phi_{t}^{0}\right\|^{2}+\frac{1}{\varepsilon}\left(f^{\prime}\left(\phi^{0}\right) \phi_{t}^{0}, \phi_{t}^{0}\right) \lesssim \varepsilon^{-\sigma_{4}}, \\
\left\|\Delta^{-1} \phi_{t t}^{0}\right\|^{2} \lesssim \varepsilon^{-\sigma_{5}}, \\
\left\|\phi_{t t}^{0}\right\|_{-1}^{2} \lesssim \varepsilon^{-\sigma_{6}} .
\end{gathered}
$$

Given Assumption 1 (i)(iii) and Assumption 2, we have following estimates for the exact solution to the Cahn-Hilliard equation. The proof is given in Appendix A.

Lemma 1 Suppose Assumption 1 (i)(iii) and Assumption 2 are satisfied. We have following regularity results for the exact solution $\phi$ of (1.1) with $\gamma=1$.

(i) $\int_{0}^{\infty}\left\|\phi_{t}\right\|_{-1}^{2} \mathrm{~d} t+\operatorname{ess} \sup E_{\varepsilon}(\phi) \lesssim \varepsilon^{-\rho_{1}}$, and $\|\phi\|_{1}^{2} \lesssim \varepsilon^{-\left(\sigma_{1}+1\right)}$;

(ii) $\operatorname{ess} \sup \left\|\phi_{t}\right\|_{-1}^{2}+\varepsilon \int_{0}^{\infty}\left\|\nabla \phi_{t}\right\|^{2} \mathrm{~d} t \lesssim \varepsilon^{-\rho_{2}}$; $t \in[0, \infty]$

(iii) ess sup $\left\|\phi_{t}\right\|^{2}+\varepsilon \int_{0}^{\infty}\left\|\Delta \phi_{t}\right\|^{2} \mathrm{~d} t \lesssim \varepsilon^{-\rho_{3}}$; $t \in[0, \infty]$

(iv) $\int_{0}^{\infty}\left\|\phi_{t t}\right\|_{-1}^{2} \mathrm{~d} t+\operatorname{ess} \sup \varepsilon\left\|\nabla \phi_{t}\right\|^{2} \lesssim \varepsilon^{-\rho_{4}}$;

(v) ess sup $\left\|\Delta^{-1} \phi_{t t}\right\|^{2}+\varepsilon \int_{0}^{\infty}\left\|\phi_{t t}\right\|^{2} \mathrm{~d} t \lesssim \varepsilon^{-\rho_{5}}$; $t \in[0, \infty]$

(vi) $\int_{0}^{\infty}\left\|\Delta^{-1} \phi_{t t t}\right\|_{-1}^{2} \mathrm{~d} t+\operatorname{ess} \sup \varepsilon\left\|\phi_{t t}\right\|_{-1}^{2} \lesssim \varepsilon^{-\rho_{6}}$;

where $\rho_{1}=\sigma_{1}$ and

$$
\begin{aligned}
& \rho_{2}=\max \left\{\sigma_{1}+3, \sigma_{2}\right\} \\
& \rho_{3}=\max \left\{\left(\sigma_{1}+1\right)(p-2)+\rho_{2}+4, \sigma_{3}\right\}, \\
& \rho_{4}=\max \left\{\rho_{2}+2+\frac{1}{2} \rho_{3}+\frac{1}{2}\left(\sigma_{1}+1\right)(p-3)^{+}, \sigma_{4}\right\}, \\
& \rho_{5}=\max \left\{\rho_{2}+\rho_{4}+1+\left(\sigma_{1}+1\right)(p-3)^{+},\left(\sigma_{1}+1\right)(p-2)+\rho_{4}+3, \sigma_{5}\right\}, \\
& \rho_{6}=\max \left\{\left(\sigma_{1}+1\right)(p-2)+\rho_{5}+3, \sigma_{6}-1\right\} .
\end{aligned}
$$


To get the convergence result of the second order schemes, we need make some assumptions on the scheme used to calculate the numerical solution at first time step.

Assumption 3 We assume that an appropriate scheme is used to calculate the numerical solution at first step, such that

$$
\begin{gathered}
m_{1}:=\frac{1}{|\Omega|} \int_{\Omega} \phi^{1}(x) \mathrm{d} x=m_{0}, \\
E_{\varepsilon}\left(\phi^{1}\right) \leq E_{\varepsilon}\left(\phi^{0}\right) \lesssim \varepsilon^{-\sigma_{1}}, \\
\frac{1}{\tau}\left\|\phi^{1}-\phi^{0}\right\|_{-1}^{2} \lesssim \varepsilon^{-\sigma_{1}}, \\
\frac{1}{\tau}\left\|\phi^{1}-\phi^{0}\right\|^{2} \lesssim \varepsilon^{-\sigma_{1}-2},
\end{gathered}
$$

and exist a constant $0<\tilde{\sigma}_{1}<\max \left\{\rho_{6}+4, \rho_{4}+6, \rho_{2}+9\right\}$ and constant $C_{1}$ independent of $\tau, \varepsilon$, such that

$$
\left\|e^{1}\right\|_{-1}^{2}+\tau \varepsilon\left\|\nabla e^{1}\right\|^{2} \leq C_{1} \varepsilon^{-\tilde{\sigma}_{1}} \tau^{4} .
$$

Following volume conservation property is easy to prove but important to the error estimate. Because of the integration of $\phi^{n}$ is conserved, $\delta_{t} \phi^{n}$ and $e^{n}$ belong to $L_{0}^{2}(\Omega)$ such that we can define $H^{-1}$ norm and use Poincare's inequality for those quantities.

Lemma 2 Suppose (3.6) and (3.13) holds, then the numerical solution of (2.14)-(2.15) satisfies

$$
\frac{1}{|\Omega|} \int_{\Omega} \phi^{n}(x) \mathrm{d} x=m_{0}, \quad n=1, \ldots, N+1,
$$

and the error function $e^{n}$ satisfies

$$
\int_{\Omega} e^{n}(x) \mathrm{d} x=0, \quad n=1, \ldots, N+1 .
$$

Now, we present our first error estimate result, which is a coarse estimate obtained by a standard approach.

Theorem 3 (Coarse error estimate) Suppose Assumption 1 (i)(ii), Assumption 2 and Assumption 3 hold. Then $\forall \tau \leq 1$, following error estimate holds for the SL-BDF2 scheme (2.14)-(2.15):

$$
\begin{aligned}
& \left\|e^{n+1}\right\|_{-1}^{2}+\left\|2 e^{n+1}-e^{n}\right\|_{-1}^{2}+2 A \tau^{2}\left\|\nabla e^{n+1}\right\|^{2} \\
& +2 A \tau^{2}\left\|\delta_{t} \nabla e^{n+1}\right\|^{2}+\tau \varepsilon\left\|\nabla e^{n+1}\right\|^{2}+\left\|\delta_{t t} e^{n+1}\right\|_{-1}^{2}+4 B \tau\left\|e^{n+1}\right\|^{2} \\
\leq & \left\|e^{n}\right\|_{-1}^{2}+\left\|2 e^{n}-e^{n-1}\right\|_{-1}^{2}+2 A \tau^{2}\left\|\nabla e^{n}\right\|^{2} \\
& +C_{2} \tau \varepsilon^{-3}\left\|2 e^{n}-e^{n-1}\right\|_{-1}^{2}+C_{3} \tau^{4} \varepsilon^{-\max \left\{\rho_{6}+1, \rho_{4}+3, \rho_{2}+6\right\}}, \quad n \geq 1,
\end{aligned}
$$

and

$$
\begin{aligned}
& \max _{1 \leq n \leq N}\left\{\left\|e^{n+1}\right\|_{-1}^{2}+\left\|2 e^{n+1}-e^{n}\right\|_{-1}^{2}+2 A \tau^{2}\left\|\nabla e^{n+1}\right\|^{2}\right\} \\
& +\sum_{n=1}^{N}\left(2 A \tau^{2}\left\|\delta_{t} \nabla e^{n+1}\right\|^{2}+\tau \varepsilon\left\|\nabla e^{n+1}\right\|^{2}+\left\|\delta_{t t} e^{n+1}\right\|_{-1}^{2}+4 B \tau\left\|e^{n+1}\right\|^{2}\right) \\
& \leq \exp \left(C_{2} \varepsilon^{-3} T\right)\left(C_{3} \varepsilon^{-\max \left\{\rho_{6}+1, \rho_{4}+3, \rho_{2}+6\right\}}+C_{1}\left(5+2 A \varepsilon^{-1} \tau\right) \varepsilon^{-\tilde{\sigma}_{1}}\right) \tau^{4},
\end{aligned}
$$

where $C_{2}, C_{3}$ are two constants that can be uniformly bounded independent of $\varepsilon$ and $\tau$. 
Proof The following equations for the error functions hold:

$$
\begin{gathered}
D_{\tau} e^{n+1}=\Delta\left(\mu^{n+1}-\mu\left(t^{n+1}\right)\right)+R_{1}^{n+1}, \\
\mu^{n+1}-\mu\left(t^{n+1}\right)=-\varepsilon \Delta e^{n+1}+\frac{1}{\varepsilon}\left[f\left(2 \phi^{n}-\phi^{n-1}\right)-f\left(\phi\left(t^{n+1}\right)\right)\right] \\
-A \tau \Delta \delta_{t} e^{n+1}+B \delta_{t t} e^{n+1}-A \Delta R_{3}^{n+1}+B R_{2}^{n+1},
\end{gathered}
$$

where the residual terms are

$$
\begin{aligned}
& R_{1}^{n+1}=\phi_{t}\left(t^{n+1}\right)-D_{\tau} \phi\left(t^{n+1}\right), \\
& R_{2}^{n+1}=\delta_{t t} \phi\left(t^{n+1}\right)=\phi\left(t^{n+1}\right)-2 \phi\left(t^{n}\right)+\phi\left(t^{n-1}\right), \\
& R_{3}^{n+1}=\tau \delta_{t} \phi\left(t^{n+1}\right)=\tau\left(\phi\left(t^{n+1}\right)-\phi\left(t^{n}\right)\right) .
\end{aligned}
$$

Pairing (3.22) with $-\Delta^{-1} e^{n+1}$, adding (3.23) paired with $-e^{n+1}$, we get

$$
\begin{aligned}
& \left(D_{\tau} e^{n+1},-\Delta^{-1} e^{n+1}\right)+\varepsilon\left\|\nabla e^{n+1}\right\|^{2}+A \tau\left(\delta_{t} \nabla e^{n+1}, \nabla e^{n+1}\right) \\
= & \left(R_{1}^{n+1},-\Delta^{-1} e^{n+1}\right)-B\left(R_{2}^{n+1}, e^{n+1}\right)-A\left(\nabla R_{3}^{n+1}, \nabla e^{n+1}\right) \\
& -B\left(\delta_{t t} e^{n+1}, e^{n+1}\right)-\frac{1}{\varepsilon}\left(f\left(2 \phi^{n}-\phi^{n-1}\right)-f\left(\phi\left(t^{n+1}\right)\right), e^{n+1}\right) \\
= & : J_{1}+J_{2}+J_{3}+J_{4}+J_{5} .
\end{aligned}
$$

First, for the terms on the left side of (3.24), we have

$$
\begin{aligned}
\left(D_{t} e^{n+1},-\Delta^{-1} e^{n+1}\right)=\frac{1}{4 \tau}\left(\left\|e^{n+1}\right\|_{-1}^{2}+\left\|2 e^{n+1}-e^{n}\right\|_{-1}^{2}\right) & \\
& -\frac{1}{4 \tau}\left(\left\|e^{n}\right\|_{-1}^{2}+\left\|2 e^{n}-e^{n-1}\right\|_{-1}^{2}\right)+\frac{1}{4 \tau}\left\|\delta_{t t} e^{n+1}\right\|_{-1}^{2},
\end{aligned}
$$

and

$$
A \tau\left(\delta_{t} \nabla e^{n+1}, \nabla e^{n+1}\right)=\frac{1}{2} A \tau\left(\left\|\nabla e^{n+1}\right\|^{2}-\left\|\nabla e^{n}\right\|^{2}+\left\|\delta_{t} \nabla e^{n+1}\right\|^{2}\right) .
$$

Then, we estimate the terms on the right hand side of (3.24).

$$
\begin{aligned}
J_{1}= & \left(R_{1}^{n+1},-\Delta^{-1} e^{n+1}\right) \leq \frac{1}{\eta_{1}}\left\|\Delta^{-1} R_{1}^{n+1}\right\|_{-1}^{2}+\frac{\eta_{1}}{4}\left\|\nabla e^{n+1}\right\|^{2} . \\
J_{2} & =-B\left(R_{2}^{n+1}, e^{n+1}\right) \leq \frac{B^{2}}{\eta_{1}}\left\|R_{2}^{n+1}\right\|_{-1}^{2}+\frac{\eta_{1}}{4}\left\|\nabla e^{n+1}\right\|^{2} . \\
J_{3} & =-A\left(\nabla R_{3}^{n+1}, \nabla e^{n+1}\right) \leq \frac{A^{2}}{\eta_{1}}\left\|\nabla R_{3}^{n+1}\right\|^{2}+\frac{\eta_{1}}{4}\left\|\nabla e^{n+1}\right\|^{2} . \\
J_{4} & =-B\left(\delta_{t t} e^{n+1}, e^{n+1}\right)=-B\left(e^{n+1}-\left(2 e^{n}-e^{n-1}\right), e^{n+1}\right) \\
& \leq-B\left\|e^{n+1}\right\|^{2}+\frac{B^{2}}{\eta_{1}}\left\|2 e^{n}-e^{n-1}\right\|_{-1}^{2}+\frac{\eta_{1}}{4}\left\|\nabla e^{n+1}\right\|^{2} . \\
J_{5} & =-\frac{1}{\varepsilon}\left(f\left(2 \phi^{n}-\phi^{n-1}\right)-f\left(\phi\left(t^{n+1}\right)\right), e^{n+1}\right) \\
& \leq \frac{L}{\varepsilon}\left(\left|2 \phi^{n}-\phi^{n-1}-\phi\left(t^{n+1}\right)\right|,\left|e^{n+1}\right|\right) \\
& =\frac{L}{\varepsilon}\left(\left|2 e^{n}-e^{n-1}-\delta_{t t} \phi\left(t^{n+1}\right)\right|,\left|e^{n+1}\right|\right) \\
\leq & \frac{L^{2}}{\varepsilon^{2} \eta_{1}}\left\|2 e^{n}-e^{n-1}\right\|_{-1}^{2}+\frac{L^{2}}{\varepsilon^{2} \eta_{1}}\left\|R_{2}^{n+1}\right\|_{-1}^{2}+\frac{\eta_{1}}{2}\left\|\nabla e^{n+1}\right\|^{2} .
\end{aligned}
$$


Combining (3.24)-(3.31) together, yields

$$
\begin{aligned}
& \frac{1}{4 \tau}\left(\left\|e^{n+1}\right\|_{-1}^{2}+\left\|2 e^{n+1}-e^{n}\right\|_{-1}^{2}\right)+\frac{1}{2} A \tau\left\|\nabla e^{n+1}\right\|^{2} \\
& +\frac{1}{2} A \tau\left\|\delta_{t} \nabla e^{n+1}\right\|^{2}+\varepsilon\left\|\nabla e^{n+1}\right\|^{2}+\frac{1}{4 \tau}\left\|\delta_{t t} e^{n+1}\right\|_{-1}^{2}+B\left\|e^{n+1}\right\|^{2} \\
\leq & \frac{1}{4 \tau}\left(\left\|e^{n}\right\|_{-1}^{2}+\left\|2 e^{n}-e^{n-1}\right\|_{-1}^{2}\right)+\frac{1}{2} A \tau\left\|\nabla e^{n}\right\|^{2} \\
& +\frac{1}{\eta_{1}}\left\|\Delta^{-1} R_{1}^{n+1}\right\|_{-1}^{2}+\left(B^{2}+\frac{L^{2}}{\varepsilon^{2}}\right) \frac{1}{\eta_{1}}\left\|R_{2}^{n+1}\right\|_{-1}^{2}+\frac{A^{2}}{\eta_{1}}\left\|\nabla R_{3}^{n+1}\right\|^{2} \\
& +\left(B^{2}+\frac{L^{2}}{\varepsilon^{2}}\right) \frac{1}{\eta_{1}}\left\|2 e^{n}-e^{n-1}\right\|_{-1}^{2}+\frac{3}{2} \eta_{1}\left\|\nabla e^{n+1}\right\|^{2} .
\end{aligned}
$$

By using Taylor expansions in integral form, one can get estimates for the residuals

$$
\begin{gathered}
\left\|\Delta^{-1} R_{1}^{n+1}\right\|_{-1}^{2} \leq c_{1} \tau^{3} \int_{t_{n-1}}^{t_{n+1}}\left\|\partial_{t t t} \Delta^{-1} \phi(t)\right\|_{-1}^{2} \mathrm{~d} t \\
\left\|R_{2}^{n+1}\right\|_{-1}^{2} \leq c_{2} \tau^{3} \int_{t_{n-1}}^{t_{n+1}}\left\|\partial_{t t} \phi(t)\right\|_{-1}^{2} \mathrm{~d} t \\
\left\|\nabla R_{3}^{n+1}\right\|^{2} \leq c_{3} \tau^{3} \int_{t_{n}}^{t_{n+1}}\left\|\partial_{t} \nabla \phi(t)\right\|^{2} \mathrm{~d} t
\end{gathered}
$$

where $c_{1}, c_{2}$ and $c_{3}$ are three constants.

Taking $\eta_{1}=\varepsilon / 2$ in (3.32) and combining the residual estimates (3.33)-(3.35) and Assumption 1,3 with equation (3.32), we get estimate (3.20) with

$$
C_{2}=8 L^{2}+8 B^{2} \varepsilon^{2}, \quad C_{3}=8 c_{1}+8 c_{2}\left(L^{2}+B^{2} \varepsilon^{2}\right)+8 c_{3} A^{2} \varepsilon^{4} .
$$

By using a discrete Gronwall inequality, we obtain (3.21).

Theorem 3 is the usual error estimate, in which the error growth depends on $1 / \varepsilon$ exponentially. To obtain a finer estimate on the error, we need to use a spectral estimate of the linearized Cahn-Hilliard operator by Chen [9] for the case when the interface is well developed in the Cahn-Hilliard system.

Lemma 3 Let $\phi(t)$ be the exact solution of Cahn-Hilliard equation (1.1) with interfaces are well developed in the initial condition (i.e. conditions (1.9)-(1.15) in [9] are satisfied). Then there exist $0<\varepsilon_{0}<1$ and positive constant $C_{0}$ such that the principle eigenvalue of the linearized CahnHilliard operator $\mathcal{L}_{C H}:=\Delta\left(\varepsilon \Delta-\frac{1}{\varepsilon} f^{\prime}(\phi) I\right)$ satisfies for all $t \in[0, T]$

$$
\lambda_{C H}=\inf _{\substack{0 \neq v \in H^{1}(\Omega) \\ \Delta \mu=v}} \frac{\varepsilon\|\nabla v\|^{2}+\frac{1}{\varepsilon}\left(f^{\prime}(\phi(\cdot, t)) v, v\right)}{\|\nabla \mu\|^{2}} \geq-C_{0},
$$

for all $\varepsilon \in\left(0, \varepsilon_{0}\right)$. 
Theorem 4 Suppose all of the Assumption 1(i)(ii),2, 3 hold. Let time step $\tau$ satisfy the following constraint

$$
\tau \leq \min \left\{\frac{1}{4\left(C_{0}+L^{2}\right)}, \frac{\varepsilon^{6}}{12\left(B^{2} \varepsilon^{2}+L^{2}\right)}, C_{5} \varepsilon^{\left(5+\frac{1}{2} \max \left\{\rho_{6}+4, \rho_{4}+6, \rho_{2}+9\right\}+\frac{d-2}{8}\right) \frac{8}{18-d}}\right\},
$$

where $C_{5}$ is a constant can be bounded uniformly independent of $\tau$ and $\varepsilon$. Then the solution of (2.14) (2.15) satisfies the following error estimate

$$
\begin{aligned}
& \max _{1 \leq n \leq N}\left\{\left\|e^{n+1}\right\|_{-1}^{2}+\left\|2 e^{n+1}-e^{n}\right\|_{-1}^{2}+2 A \tau^{2}\left\|\nabla e^{n+1}\right\|^{2}\right\} \\
&+\sum_{n=1}^{N}\left(2 A \tau^{2}\left\|\delta_{t} \nabla e^{n+1}\right\|^{2}+\tau \frac{\varepsilon^{4}}{2}\left\|\nabla e^{n+1}\right\|^{2}\right) \\
& \lesssim \varepsilon^{-\max \left\{\rho_{6}+4, \rho_{4}+6, \rho_{2}+9\right\}} \tau^{4} .
\end{aligned}
$$

Proof We refine the result of Theorem 3 by re-estimating $J_{4}$ in equation (3.24) as

$$
J_{4}=-B\left(\delta_{t t} e^{n+1}, e^{n+1}\right) \leq \frac{B^{2}}{\eta_{1}}\left\|\delta_{t t} e^{n+1}\right\|_{-1}^{2}+\frac{\eta_{1}}{4}\left\|\nabla e^{n+1}\right\|^{2},
$$

and rewriting $J_{5}$ as

$$
\begin{aligned}
J_{5} & =J_{6}+J_{7}, \\
J_{6} & =-\frac{1}{\varepsilon}\left(f\left(2 \phi^{n}-\phi^{n-1}\right)-f\left(\phi^{n+1}\right), e^{n+1}\right) \\
& \leq \frac{L}{\varepsilon}\left(\left|\delta_{t t} e^{n+1}\right|+\left|R_{2}^{n+1}\right|,\left|e^{n+1}\right|\right) \\
& \leq \frac{L^{2}}{\varepsilon^{2} \eta_{1}}\left(\left\|\delta_{t t} e^{n+1}\right\|_{-1}^{2}+\left\|R_{2}^{n+1}\right\|_{-1}^{2}\right)+\frac{\eta_{1}}{2}\left\|\nabla e^{n+1}\right\|^{2}, \\
J_{7} & =-\frac{1}{\varepsilon}\left(f\left(\phi^{n+1}\right)-f\left(\phi\left(t^{n+1}\right)\right), e^{n+1}\right) \\
& \leq-\frac{1}{\varepsilon}\left(f^{\prime}\left(\phi\left(t^{n+1}\right)\right) e^{n+1}, e^{n+1}\right)+\frac{L_{2}}{\varepsilon}\left\|e^{n+1}\right\|_{L^{3}}^{3} .
\end{aligned}
$$

The spectrum estimate (3.36) give us

$$
\varepsilon\left\|\nabla e^{n+1}\right\|^{2}+\frac{1}{\varepsilon}\left(f^{\prime}\left(\phi\left(t^{n+1}\right)\right) e^{n+1}, e^{n+1}\right) \geq-C_{0}\left\|e^{n+1}\right\|_{-1}^{2} .
$$

If the entire $\varepsilon\left\|\nabla e^{n+1}\right\|^{2}$ term is used to control the the term involving $f^{\prime}$ in $J_{7}$, we will not be able to control the $\left\|\nabla e^{n+1}\right\|$ terms in $J_{1}, \ldots, J_{4}$ and $J_{6}$. So we apply (3.43) with a scaling factor $(1-\eta)$ close to but smaller than 1 , to get

$$
-(1-\eta) \frac{1}{\varepsilon}\left(f^{\prime}\left(\phi\left(t^{n+1}\right)\right) e^{n+1}, e^{n+1}\right) \leq(1-\eta) C_{0}\left\|e^{n+1}\right\|_{-1}^{2}+(1-\eta) \varepsilon\left\|\nabla e^{n+1}\right\|^{2} .
$$

On the other hand,

$$
-\frac{\eta}{\varepsilon}\left(f^{\prime}\left(\phi\left(t^{n+1}\right)\right) e^{n+1}, e^{n+1}\right) \leq \frac{L^{2}}{\varepsilon^{2}} \frac{\eta}{\eta_{2}}\left\|e^{n+1}\right\|_{-1}^{2}+\frac{\eta \eta_{2}}{4}\left\|\nabla e^{n+1}\right\|^{2} .
$$

Combining (3.42), (3.44) and (3.45) together, we have

$$
J_{7} \leq\left(C_{0}(1-\eta)+\frac{L^{2}}{\varepsilon^{2}} \frac{\eta}{\eta_{2}}\right)\left\|e^{n+1}\right\|_{-1}^{2}+\frac{L_{2}}{\varepsilon}\left\|e^{n+1}\right\|_{L^{3}}^{3}+\left((1-\eta) \varepsilon+\frac{\eta \eta_{2}}{4}\right)\left\|\nabla e^{n+1}\right\|^{2} .
$$


Substituting the estimate of (3.25)-(3.29), (3.39)-(3.41) and (3.46) into (3.24), we get

$$
\begin{aligned}
& \frac{1}{4 \tau}\left(\left\|e^{n+1}\right\|_{-1}^{2}+\left\|2 e^{n+1}-e^{n}\right\|_{-1}^{2}\right)+\frac{1}{2} A \tau\left\|\nabla e^{n+1}\right\|^{2} \\
& +\frac{1}{2} A \tau\left\|\delta_{t} \nabla e^{n+1}\right\|^{2}+\varepsilon\left\|\nabla e^{n+1}\right\|^{2}+\frac{1}{4 \tau}\left\|\delta_{t t} e^{n+1}\right\|_{-1}^{2} \\
\leq & \frac{1}{4 \tau}\left(\left\|e^{n}\right\|_{-1}^{2}+\left\|2 e^{n}-e^{n-1}\right\|_{-1}^{2}\right)+\frac{1}{2} A \tau\left\|\nabla e^{n}\right\|^{2} \\
& +\frac{1}{\eta_{1}}\left\|\Delta^{-1} R_{1}^{n+1}\right\|_{-1}^{2}+\left(B^{2}+\frac{L^{2}}{\varepsilon^{2}}\right) \frac{1}{\eta_{1}}\left\|R_{2}^{n+1}\right\|_{-1}^{2}+\frac{A^{2}}{\eta_{1}}\left\|\nabla R_{3}^{n+1}\right\|^{2} \\
& +\left(B^{2}+\frac{L^{2}}{\varepsilon^{2}}\right) \frac{1}{\eta_{1}}\left\|\delta_{t t} e^{n+1}\right\|_{-1}^{2}+\frac{3}{2} \eta_{1}\left\|\nabla e^{n+1}\right\|^{2} \\
& +\left(C_{0}(1-\eta)+\frac{L^{2}}{\varepsilon^{2}} \frac{\eta}{\eta_{2}}\right)\left\|e^{n+1}\right\|_{-1}^{2}+\frac{L_{2}}{\varepsilon}\left\|e^{n+1}\right\|_{L^{3}}^{3}+\left((1-\eta) \varepsilon+\frac{\eta \eta_{2}}{4}\right)\left\|\nabla e^{n+1}\right\|^{2} .
\end{aligned}
$$

We now estimate the $L^{3}$ term. By interpolating $L^{3}$ between $L^{2}$ and $H^{1}$ and using Poincare's inequality for error function, we get

$$
\left\|e^{n+1}\right\|_{L^{3}}^{3} \leq K\left\|\nabla e^{n+1}\right\|^{\frac{d}{2}}\left\|e^{n+1}\right\|^{\frac{6-d}{2}}
$$

where $K$ is a constant independent of $\varepsilon$ and $\tau$. We continue the estimate by using $\left\|e^{n+1}\right\|^{2} \leq$ $\left\|\nabla e^{n+1}\right\| \cdot\left\|e^{n+1}\right\|_{-1}$ to get

$$
\frac{L_{2}}{\varepsilon}\left\|e^{n+1}\right\|_{L^{3}}^{3} \leq L_{2} K \varepsilon^{-1}\left\|\nabla e^{n+1}\right\|^{\frac{d}{2}+\frac{6-d}{4}}\left\|e^{n+1}\right\|_{-1}^{\frac{6-d}{4}}=G^{n+1}\left\|\nabla e^{n+1}\right\|^{2},
$$

where $G^{n+1}=L_{2} K \varepsilon^{-1}\left\|\nabla e^{n+1}\right\|^{\frac{d-2}{4}}\left\|e^{n+1}\right\|_{-1}^{\frac{6-d}{4}}$.

Now plug equation (3.48) into (3.47), and take $\eta_{2}=\varepsilon, \eta=\varepsilon^{3}, \eta_{1}=\varepsilon^{4} / 3$ and $\tau \leq$ $\varepsilon^{6} / 12\left(B^{2} \varepsilon^{2}+L^{2}\right)$, such that

$$
\frac{3 \eta_{1}}{2}+(1-\eta) \varepsilon+\frac{\eta \eta_{2}}{4}=\varepsilon-\frac{1}{4} \varepsilon^{4}, \quad\left(B^{2}+\frac{L^{2}}{\varepsilon^{2}}\right) \frac{1}{\eta_{1}} \leq \frac{1}{4 \tau}, \quad \frac{L^{2}}{\varepsilon^{2}} \frac{\eta}{\eta_{2}}=L^{2},
$$

we get

$$
\begin{aligned}
& \frac{1}{4 \tau}\left(\left\|e^{n+1}\right\|_{-1}^{2}+\left\|2 e^{n+1}-e^{n}\right\|_{-1}^{2}\right)+\frac{1}{2} A \tau\left\|\nabla e^{n+1}\right\|^{2} \\
& +\frac{1}{2} A \tau\left\|\delta_{t} \nabla e^{n+1}\right\|^{2}+\frac{\varepsilon^{4}}{4}\left\|\nabla e^{n+1}\right\|^{2} \\
\leq & \frac{1}{4 \tau}\left(\left\|e^{n}\right\|_{-1}^{2}+\left\|2 e^{n}-e^{n-1}\right\|_{-1}^{2}\right)+\frac{1}{2} A \tau\left\|\nabla e^{n}\right\|^{2} \\
& +\frac{3}{\varepsilon^{4}}\left\|\Delta^{-1} R_{1}^{n+1}\right\|_{-1}^{2}+\left(B^{2}+\frac{L^{2}}{\varepsilon^{2}}\right) \frac{3}{\varepsilon^{4}}\left\|R_{2}^{n+1}\right\|_{-1}^{2}+\frac{3 A^{2}}{\varepsilon^{4}}\left\|\nabla R_{3}^{n+1}\right\|^{2} \\
& +\left(C_{0}(1-\eta)+L^{2}\right)\left\|e^{n+1}\right\|_{-1}^{2}+G^{n+1}\left\|\nabla e^{n+1}\right\|^{2} .
\end{aligned}
$$

If $G^{n+1}$ is uniformly bounded by constant $\varepsilon^{4} / 8$, then we can get a finer error estimate for $\tau<1 / 8\left(C_{0}+L^{2}\right)$ by using discrete Gronwall inequality and the assumption of first step error 
(3.17):

$$
\begin{gathered}
\max _{1 \leq n \leq N}\left\{\left\|e^{n+1}\right\|_{-1}^{2}+\left\|2 e^{n+1}-e^{n}\right\|_{-1}^{2}+2 A \tau^{2}\left\|\nabla e^{n+1}\right\|^{2}\right\} \\
+\sum_{n=1}^{N}\left(2 A \tau^{2}\left\|\delta_{t} \nabla e^{n+1}\right\|^{2}+\tau \varepsilon^{4} / 2\left\|\nabla e^{n+1}\right\|^{2}\right) \\
\leq \frac{3}{2} C_{3}^{\prime} \exp \left(8\left(C_{0}+L^{2}\right) T\right) \varepsilon^{-\max \left\{\rho_{6}+4, \rho_{4}+6, \rho_{2}+9\right\}} \tau^{4}, \quad N \geq 1,
\end{gathered}
$$

where $C_{3}^{\prime}=C_{3}+C_{1}\left(5+2 A \varepsilon^{-1} \tau\right)$. We prove this by induction. Assuming that the above estimate holds for all first $N-1$ time steps. Then the coarse estimate (3.20) leads to

$$
\begin{aligned}
\left\|e^{N+1}\right\|_{-1}^{2}+\tau \varepsilon\left\|\nabla e^{N+1}\right\|^{2} \leq & \left\|e^{N}\right\|_{-1}^{2}+\left(1+C_{2} \tau \varepsilon^{-3}\right)\left\|2 e^{N}-e^{N-1}\right\|_{-1}^{2} \\
& +2 A \tau^{2}\left\|\nabla e^{N}\right\|^{2}+C_{3} \tau^{4} \varepsilon^{-\max \left\{\rho_{6}+1, \rho_{4}+3, \rho_{2}+6\right\}} .
\end{aligned}
$$

Then by induction assumption (3.50), we get

$$
\left\|e^{N+1}\right\|_{-1}^{2}+\tau \varepsilon\left\|\nabla e^{N+1}\right\|^{2} \leq C_{4} \varepsilon^{-\max \left\{\rho_{6}+4, \rho_{4}+6, \rho_{2}+9\right\}} \tau^{4}
$$

where $C_{4}=\frac{3}{2} C_{3}^{\prime}\left(1+C_{2} \tau \varepsilon^{-3}\right) \exp \left(8\left(C_{0}+L^{2}\right) T\right)+C_{3} \varepsilon^{3}$. Thus, if

$$
L_{2} K \varepsilon^{-1}\left[C_{4} \varepsilon^{-\max \left\{\rho_{6}+4, \rho_{4}+6, \rho_{2}+9\right\}-1} \tau^{3}\right]^{\frac{d-2}{8}}\left[C_{4} \varepsilon^{-\max \left\{\rho_{6}+4, \rho_{4}+6, \rho_{2}+9\right\}} \tau^{4}\right]^{\frac{6-d}{8}} \leq \varepsilon^{4} / 8,
$$

we get $G^{N+1} \leq \varepsilon^{4} / 8$. Solving (3.53), we get

$$
\tau \leq C_{5} \varepsilon^{\left(5+\frac{1}{2} \max \left\{\rho_{6}+4, \rho_{4}+6, \rho_{2}+9\right\}+\frac{d-2}{8}\right) \frac{8}{18-d},}
$$

where $C_{5}=\left(8 L_{2} K C_{4}^{\frac{1}{2}}\right)^{-\frac{8}{18-d}}$. The proof is completed.

Remark 4 Different to the related work [18], we did not take $\eta=\tau^{\beta}$ in equation (3.47), this allows us to maintain the full order about $\tau$. Besides, our induction method to handle $L^{3}$ term is much simpler than the method used in [18] and [30], where first-order schemes are studied.

Remark 5 Theorem 4 and its proof is valid for the special cases $A=0$ and/or $B=0$, since the condition (2.17) is not used in the proof.

\section{Implementation and numerical results}

In this section, we numerically verify our schemes are second order accurate in time and energy stable.

We use the commonly used double-well potential $F(\phi)=\frac{1}{4}\left(\phi^{2}-1\right)^{2}$. It is a common practice to modify $F(\phi)$ to have a quadratic growth for $|\phi|>1$ (since physically $|\phi| \leq 1$ ), such that a global Lipschitz condition is satisfied [38], [10]. To get a $C^{4}$ smooth double-well potential with quadratic growth, we introduce $\tilde{F}(\phi) \in C^{\infty}(\mathbf{R})$ as a smooth mollification of

$$
\hat{F}(\phi)= \begin{cases}\frac{11}{2}(\phi-2)^{2}+6(\phi-2)+\frac{9}{4}, & \phi>2, \\ \frac{1}{4}\left(\phi^{2}-1\right)^{2}, & \phi \in[-2,2], \\ \frac{11}{2}(\phi+2)^{2}+6(\phi+2)+\frac{9}{4}, & \phi<-2 .\end{cases}
$$

with a mollification parameter much smaller than 1 , to replace $F(\phi)$. Note that the truncation points -2 and 2 used here are for convenience only. Other values outside of region $[-1,1]$ can be used as well. For simplicity, we still denote the modified function $\tilde{F}$ by $F$. 

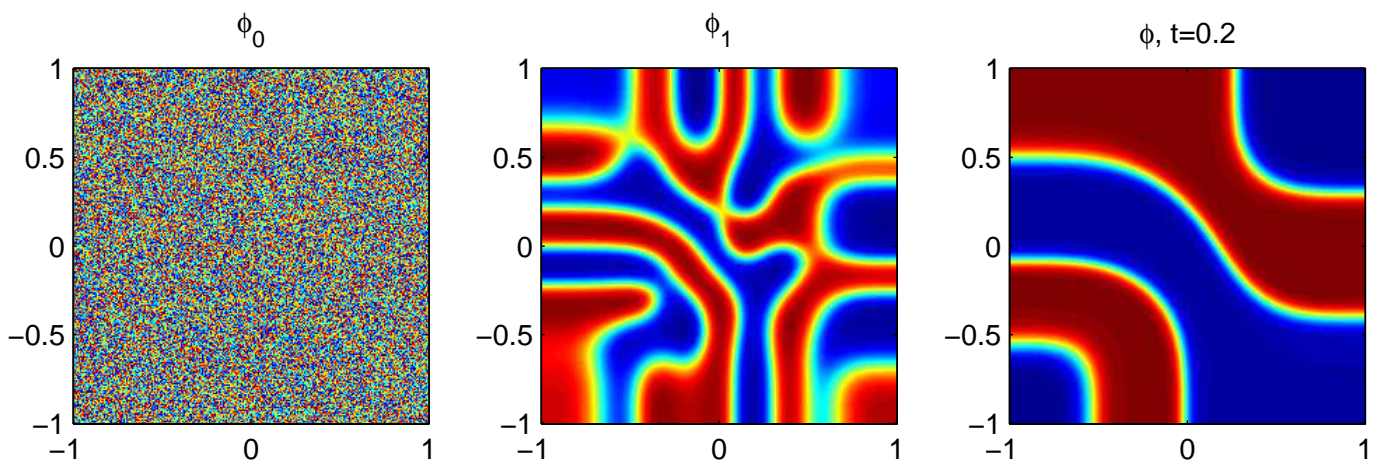

Fig. 1 The two random initial values $\phi_{0}, \phi_{1}$ and the state of $\phi_{1}$ evolves 0.2 time unit according to the Cahn-Hilliard equation (1.1) with $\gamma=1$.

\subsection{Space discrete and implementation}

To test the numerical scheme, we solve (1.1) in a 2-dimensional tensor product domain $\Omega=[-1,1] \times[-1,1]$. We use a Legendre Galerkin method similar as in [39,49] for spatial discretization. Let $L_{k}(x)$ denote the Legendre polynomial of degree $k$. We define

$$
V_{M}=\operatorname{span}\left\{\varphi_{k}(x) \varphi_{j}(y), k, j=0, \ldots, M-1\right\} \in H^{1}(\Omega),
$$

where $\varphi_{0}(x)=L_{0}(x) ; \varphi_{1}(x)=L_{1}(x) ; \varphi_{k}(x)=L_{k}(x)-L_{k+2}(x), k=2, \ldots, M-1$, be the Galerkin approximation space for both $\phi^{n+1}$ and $\mu^{n+1}$. Then the full discretized form for the SL-BDF2 scheme reads:

Find $\left(\phi^{n+1}, \mu^{n+1}\right) \in\left(V_{M}\right)^{2}$ such that

$$
\begin{gathered}
\frac{1}{2 \tau}\left(3 \phi^{n+1}-4 \phi^{n}+\phi^{n-1}, \omega\right)=-\gamma\left(\nabla \mu^{n+1}, \nabla \omega\right), \quad \forall \omega \in V_{M}, \\
\left(\mu^{n+1}, \varphi\right)=\varepsilon\left(\nabla \phi^{n+1}, \nabla \varphi\right)+\frac{1}{\varepsilon}\left(f\left(2 \phi^{n}-\phi^{n-1}\right), \varphi\right)+A \tau\left(\nabla \delta_{t} \phi^{n+1}, \nabla \varphi\right) \\
+B\left(\delta_{t t} \phi^{n+1}, \varphi\right), \quad \forall \varphi \in V_{M} .
\end{gathered}
$$

This is a linear system with constant coefficients for $\left(\phi^{n+1}, \mu^{n+1}\right)$, which can be efficiently solved. We use a spectral transform with double quadrature points to eliminate the aliasing error and efficiently evaluate the integration $\left(f\left(2 \phi^{n}-\phi^{n-1}\right), \varphi\right)$ in equation (4.3).

We take $\varepsilon=0.05$ and $M=127$ and use two different initial values to test the stability and accuracy of the proposed schemes:

(i) $\left\{\phi_{0}\left(x_{i}, y_{j}\right)\right\} \in \mathbf{R}^{2 M \times 2 M}$ with $x_{i}, y_{j}$ are tensor product Legendre-Gauss quadrature points and $\phi_{0}\left(x_{i}, y_{j}\right)$ is a uniformly distributed random number between -1 and 1 (shown in the left picture of Fig. 1);

(ii) The solution of the Cahn-Hilliard equation at $t=64 \varepsilon^{3}$ which takes $\phi_{0}$ as its initial value (Denoted by $\phi_{1}$ shown in the middle picture of Fig. 1).

Given $\phi_{0}$, to start the second order schemes, we use following first order stabilized scheme to generate $\phi^{1}$

$$
\frac{\varphi^{n+1}-\varphi^{n}}{s}=\Delta \omega^{n+1}, \quad \omega^{n+1}=-\varepsilon \Delta \varphi^{n+1}+\frac{1}{\varepsilon} f\left(\varphi^{n}\right)+S \delta_{t} \varphi^{n+1}, \quad n=0, \ldots, m-1,
$$




\begin{tabular}{|c|c|c|c|c|c|c|c|c|}
\hline \multirow{2}{*}{$\tau$} & \multicolumn{4}{|c|}{ SL-BDF2 } & \multicolumn{4}{c|}{ SL-CN } \\
\cline { 2 - 9 } & \multicolumn{2}{|c|}{$\gamma=0.0025$} & \multicolumn{2}{|c|}{$\gamma=1$} & \multicolumn{2}{c|}{$\gamma=0.0025$} & \multicolumn{2}{c|}{$\gamma=1$} \\
\cline { 2 - 9 } & $B=0$ & $B=10$ & $B=0$ & $B=10$ & $B=0$ & $B=10$ & $B=0$ & $B=10$ \\
\hline 10 & 1 & 0.5 & 12.5 & 12.5 & 1 & 0.25 & 12.5 & 12.5 \\
\hline 1 & 2 & 0.5 & 25 & 25 & 1 & 0.5 & 25 & 12.5 \\
\hline 0.1 & 1 & 0.25 & 200 & 100 & 1 & 0.25 & 200 & 50 \\
\hline 0.01 & 0 & 0 & 400 & 200 & 1 & 0.25 & 400 & 100 \\
\hline 0.001 & 0 & 0 & 800 & 200 & 0 & 0 & 400 & 100 \\
\hline 0.0001 & 0 & 0 & 200 & 0 & 0 & 0 & 400 & 100 \\
\hline $1 \mathrm{E}-05$ & 0 & 0 & 0 & 0 & 0 & 0 & 400 & 50 \\
\hline $1 \mathrm{E}-06$ & 0 & 0 & 0 & 0 & 0 & 0 & 0 & 0 \\
\hline
\end{tabular}

Table 1 The minimum values of $A$ (only values $\left\{0,2^{i}, i=-7, \ldots, 1\right\} \times 4 \gamma / \varepsilon^{2}$ are tested) to make SL-BDF2 and SL-CN scheme stable when $\gamma, B$ and $\tau$ taking different values.

\begin{tabular}{|c|c|c|c|c|c|c|c|c|}
\hline \multirow{2}{*}{$\tau$} & \multicolumn{4}{|c|}{ SL-BDF2 } & \multicolumn{4}{c|}{ SL-CN } \\
\cline { 2 - 9 } & \multicolumn{2}{|c|}{$\gamma=0.0025$} & \multicolumn{2}{c|}{$\gamma=1$} & \multicolumn{2}{c|}{$\gamma=0.0025$} & \multicolumn{2}{c|}{$\gamma=1$} \\
\cline { 2 - 9 } & $A=0$ & $A=0.0625$ & $A=0$ & $A=25$ & $A=0$ & $A=0.0625$ & $A=0$ & $A=25$ \\
\hline 10 & 320 & 40 & $>640$ & 0 & 320 & 20 & $>640$ & 0 \\
\hline 1 & 40 & 40 & $>640$ & 0 & 80 & 20 & $>640$ & 0 \\
\hline 0.1 & 20 & 20 & $>640$ & 40 & 20 & 20 & 640 & 20 \\
\hline 0.01 & 0 & 0 & 40 & 40 & 20 & 20 & 320 & 20 \\
\hline 0.001 & 0 & 0 & 40 & 40 & 0 & 0 & 40 & 20 \\
\hline 0.0001 & 0 & 0 & 10 & 10 & 0 & 0 & 20 & 20 \\
\hline $1 \mathrm{E}-05$ & 0 & 0 & 0 & 0 & 0 & 0 & 20 & 20 \\
\hline $1 \mathrm{E}-06$ & 0 & 0 & 0 & 0 & 0 & 0 & 0 & 0 \\
\hline
\end{tabular}

Table 2 The minimum values of $B$ (only values $\left\{0,2^{i}, i=-3, \ldots, 4\right\} \times 2 / \varepsilon$ are tested) to make scheme SL-BDF2 and SL-CN stable when $\gamma, A$ and $\tau$ taking different values.

where $S=1 / \varepsilon$ is a stabilization constant, $s$ is the time step-size, $\varphi^{0}=\phi_{0}$. To get an accurate $\phi^{1}$, we let $s=\tau / m$ with $m=10$, and let $\phi^{1}=\varphi^{m}$.

\subsection{Stability results}

Table 1,2 show the required minimum values of $A$ (resp. $B$ ) with different $\gamma, B$ (resp. $A$ ) and $\tau$ values for stably solving (the increase of discrete energy in each time-step is less than $10^{-10}$ for 1024 time steps) the Cahn-Hilliard equation (1.1) with initial value $\phi_{0}$. The results for the initial value $\phi_{1}$ are similar. From the two tables, we observe that:

1. For smaller $\tau$ values, the SL-BDF2 scheme need smaller $A, B$ to be stable comparing to the SL-CN scheme, while both of them are stable with $A=0, B=0$ when $\tau$ is small enough. On the other hand, for larger $\tau$, SL-BDF2 schemes need relatively larger $A$ and $B$ than SL-CN scheme. This might due to the fact that the SL-BDF2 scheme has larger diffusion and splitting error than SL-CN scheme.

2. The existence of a non-zero $B$ remarkably reduce the values of $A$ needed for the scheme to be energy stable, especially when $\gamma=1$ and $10^{-5} \leq \tau \leq 0.1$. On the other hand, a non-zero $A$ remarkably reduce the values of $B$ needed for the scheme to be energy stable when $\tau$ is large.

Figure 2 presents the discrete energy dissipation of the SL-CN and SL-BDF2 scheme using several time step-sizes. We see the energy decaying property is maintained. 

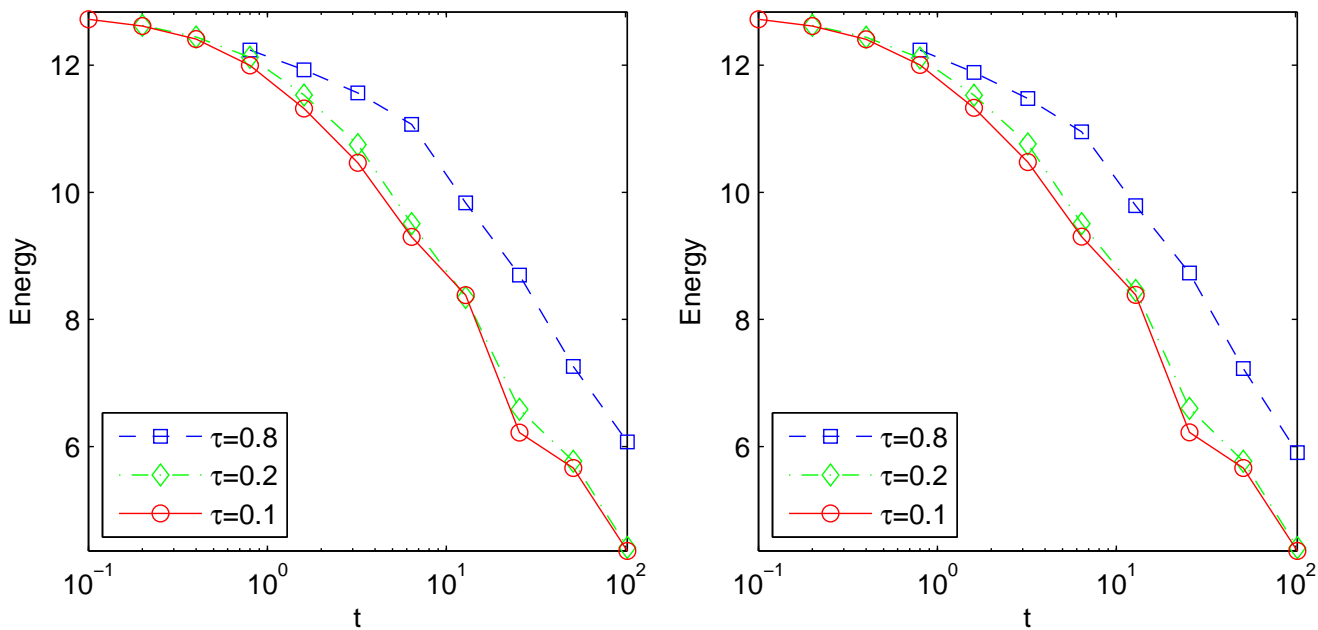

Fig. 2 The discrete energy dissipation of the two schemes solving the Cahn-Hilliard equation with initial value $\phi_{1}$, and relaxation parameter $\gamma=0.0025$. Stability constant $A=0.25, B=20$ are used. Left) result of SL-CN scheme; Right) result of SL-BDF2 scheme.

\begin{tabular}{|c|c|c|c|c|c|c|}
\hline$\tau$ & $H^{-1}$ Error & Order & $L^{2}$ Error & Order & $H^{1}$ Error & Order \\
\hline 0.16 & $3.32 \mathrm{E}-02$ & & $2.63 \mathrm{E}-01$ & & $3.49 \mathrm{E}+00$ & \\
\hline 0.08 & $9.71 \mathrm{E}-03$ & 1.77 & $8.02 \mathrm{E}-02$ & 1.72 & $1.10 \mathrm{E}+00$ & 1.67 \\
\hline 0.04 & $2.54 \mathrm{E}-03$ & 1.94 & $2.10 \mathrm{E}-02$ & 1.93 & $2.89 \mathrm{E}-01$ & 1.93 \\
\hline 0.02 & $6.38 \mathrm{E}-04$ & 1.99 & $5.25 \mathrm{E}-03$ & 2.00 & $7.21 \mathrm{E}-02$ & 2.00 \\
\hline 0.01 & $1.58 \mathrm{E}-04$ & 2.02 & $1.30 \mathrm{E}-03$ & 2.02 & $1.78 \mathrm{E}-02$ & 2.02 \\
\hline 0.005 & $3.80 \mathrm{E}-05$ & 2.05 & $3.14 \mathrm{E}-04$ & 2.05 & $4.30 \mathrm{E}-03$ & 2.05 \\
\hline
\end{tabular}

Table 3 The convergence of the SL-BDF2 scheme with $B=40, A=0.25$ for the Cahn-Hilliard equation with initial value $\phi_{1}$, parameter $\gamma=0.0025$. The errors are calculated at $T=12.8$ for both schemes.

\begin{tabular}{|c|c|c|c|c|c|c|}
\hline$\tau$ & $H^{-1}$ Error & Order & $L^{2}$ Error & Order & $H^{1}$ Error & Order \\
\hline 0.16 & $3.26 \mathrm{E}-02$ & & $2.58 \mathrm{E}-01$ & & $3.42 \mathrm{E}+00$ & \\
\hline 0.08 & $9.32 \mathrm{E}-03$ & 1.81 & $7.64 \mathrm{E}-02$ & 1.76 & $1.04 \mathrm{E}+00$ & 1.71 \\
\hline 0.04 & $2.41 \mathrm{E}-03$ & 1.95 & $1.98 \mathrm{E}-02$ & 1.95 & $2.71 \mathrm{E}-01$ & 1.95 \\
\hline 0.02 & $6.06 \mathrm{E}-04$ & 1.99 & $4.96 \mathrm{E}-03$ & 2.00 & $6.77 \mathrm{E}-02$ & 2.00 \\
\hline 0.01 & $1.50 \mathrm{E}-04$ & 2.01 & $1.23 \mathrm{E}-03$ & 2.01 & $1.68 \mathrm{E}-02$ & 2.01 \\
\hline 0.005 & $3.64 \mathrm{E}-05$ & 2.05 & $2.97 \mathrm{E}-04$ & 2.05 & $4.06 \mathrm{E}-03$ & 2.05 \\
\hline
\end{tabular}

Table 4 The convergence of the SL-CN scheme with $B=20, A=0.25$ for the Cahn-Hilliard equation with initial value $\phi_{1}$, parameter $\gamma=0.0025$. The errors are calculated at $T=12.8$ for both schemes.

\subsection{Accuracy results}

We take initial value $\phi_{1}$ to test the accuracy of the two schemes. The Cahn-Hilliard equation with $\gamma=0.0025$ are solved from $t=0$ to $T=12.8$. To calculate the numerical error, we use the numerical result generated using $\tau=10^{-3}$ as a reference of exact solution. The results are given in Table 3 and Table 4 . We see that the schemes are both second order accuracy in $H^{-1}, L^{2}$ and $H^{1}$ norm. 


\section{Conclusions}

We propose two second order stabilized linear schemes (SL-BDF2 scheme and SL-CN scheme) for the phase-field Cahn-Hilliard equation. In both schemes, the nonlinear bulk forces are treated explicitly with two additional linear stabilization terms: $-A \tau \Delta \delta_{t} \phi^{n+1}$ and $B \delta_{t t} \phi^{n+1}$. In particular, the introduction of a $H^{1}$ stabilization term $A \tau \Delta \delta_{t} \phi^{n+1}$ enables us to prove the unconditionally stability results. We also give a rigorous optimal error analysis of the SLBDF2 scheme. This error analysis holds for the special case $A=0$ and/or $B=0$ as well. Numerical results are presented to verify the stability and accuracy of the proposed schemes. By combining nonzero values of $B$ and $A$, the two schemes can obtain better stability results than the cases use only one stabilization term.

\section{Acknowledgment}

The authors would like to thank Prof. Jie Shen and Prof. Xiaobing Feng for helpful discussions. This work is partially supported by NNSFC under Grant 11771439, 11371358, 91530322.

\section{Appendix: Proof of Lemma 1}

Proof We first write down some inequalities that will be frequently used. The first one is the Holder's inequality

$$
\|u v w\|_{L^{s}} \leq\|u\|_{L^{p}}\|v\|_{L^{q}}\|w\|_{L^{r}}, \quad \forall p, q, r \in(0, \infty], \frac{1}{s}=\frac{1}{p}+\frac{1}{q}+\frac{1}{r} .
$$

The second one is the Sobolev inequality

$$
\|u\|_{L^{q}} \leq C_{s}\|u\|_{1}
$$

where $q \in[2, \infty)$ for $d=2 ; q \in\left[2, \frac{2 d}{d-2}\right]$ for $d>2 ; C_{s}$ is a general constant independent of $\phi$. We can further use Poincare's inequality to get

$$
\|v\|_{L^{q}} \leq C_{s}\|\nabla v\|, \quad \forall v \in L_{0}^{2}(\Omega) .
$$

For $v \in L_{0}^{2}(\Omega)$, we also have following inequality

$$
\|v\|^{2}=\left(\nabla v, \nabla(-\Delta)^{-1} v\right) \leq \frac{1}{2 \delta}\|\nabla v\|^{2}+\frac{\delta}{2}\|v\|_{-1}^{2},
$$

where $\delta>0$ is an arbitrary constant.

Now, we begin the proof.

(i) When $\gamma=1$, we have Cahn-Hilliard equation

$$
\phi_{t}+\varepsilon \Delta^{2} \phi=\frac{1}{\varepsilon} \Delta f(\phi) .
$$

Multiplying (A.5) by $-\Delta^{-1} \phi_{t}$ and using integration by parts, we get

$$
\left\|\phi_{t}\right\|_{-1}^{2}+\frac{\varepsilon}{2} \frac{d}{d t}\|\nabla \phi\|^{2}=-\frac{1}{\varepsilon}\left(f(\phi), \phi_{t}\right)=-\frac{1}{\varepsilon} \frac{d}{d t} \int_{\Omega} F(\phi) d x .
$$

After integrating over $[0, T]$, we obtain

$$
\int_{0}^{T}\left\|\phi_{t}\right\|_{-1}^{2} \mathrm{~d} t+E_{\varepsilon}(\phi(T))=E_{\varepsilon}\left(\phi^{0}\right)
$$


Taking maximum values of terms on the left hand side for $T \in[0, \infty]$, we get the first part of (i) from (3.7). From the definition of $E_{\varepsilon}(\phi)$, and assumption (3.1) we know

$$
\|\phi\|_{L^{2}}^{2} \leq B_{0}|\Omega|+B_{1} \varepsilon^{-\sigma_{1}+1} \lesssim \varepsilon^{-\left(\sigma_{1}-1\right)^{+}} .
$$

Combining above estimate with the fact $\frac{\varepsilon}{2}\|\nabla \phi\|^{2} \lesssim \varepsilon^{-\sigma_{1}}$, we get

$$
\|\phi\|_{1}^{2} \lesssim \varepsilon^{-\left(\sigma_{1}+1\right)} .
$$

(ii) We formally differentiate (A.5) in time to obtain

$$
\phi_{t t}+\varepsilon \Delta^{2} \phi_{t}=\frac{1}{\varepsilon} \Delta\left(f^{\prime}(\phi) \phi_{t}\right) .
$$

Pairing (A.10) with $-\Delta^{-1} \phi_{t}$ and using (A.4), yields

$$
\begin{aligned}
\frac{1}{2} \frac{d}{d t}\left\|\phi_{t}\right\|_{-1}^{2}+\varepsilon\left\|\nabla \phi_{t}\right\|^{2} & =-\frac{1}{\varepsilon}\left(f^{\prime}(\phi) \phi_{t}, \phi_{t}\right) \leq \frac{\tilde{c}_{0}}{\varepsilon}\left\|\phi_{t}\right\|^{2} \\
& \leq \frac{\varepsilon}{2}\left\|\nabla \phi_{t}\right\|^{2}+\frac{\tilde{c}_{0}^{2}}{2 \varepsilon^{3}}\left\|\phi_{t}\right\|_{-1}^{2} .
\end{aligned}
$$

Integrating (A.11) over $[0, T]$ and taking maximum values for terms depending on $T$, we get

$$
\underset{t \in[0, \infty]}{\operatorname{ess~sup}}\left\|\phi_{t}\right\|_{-1}^{2}+\varepsilon \int_{0}^{\infty}\left\|\nabla \phi_{t}\right\|^{2} \mathrm{~d} t \lesssim \frac{\tilde{c}_{0}^{2}}{\varepsilon^{3}} \int_{0}^{\infty}\left\|\phi_{t}\right\|_{-1}^{2} \mathrm{~d} t+\left\|\phi_{t}^{0}\right\|_{-1}^{2} .
$$

The assertion then follows from (i) and the inequality (3.8) of Assumption 2.

(iii) Testing (A.10) with $\phi_{t}$, using (A.1) and (A.2) with Poincare's inequality, we get

$$
\begin{aligned}
\frac{1}{2} \frac{d}{d t}\left\|\phi_{t}\right\|^{2}+\varepsilon\left\|\Delta \phi_{t}\right\|^{2} & =\frac{1}{\varepsilon}\left(f^{\prime}(\phi) \phi_{t}, \Delta \phi_{t}\right) \leq \frac{1}{\varepsilon}\left\|f^{\prime}(\phi)\right\|_{L^{3}}\left\|\phi_{t}\right\|_{L^{6}}\left\|\Delta \phi_{t}\right\| \\
& \leq \frac{\varepsilon}{2}\left\|\Delta \phi_{t}\right\|^{2}+\frac{1}{2 \varepsilon^{3}}\left\|f^{\prime}(\phi)\right\|_{L^{3}}^{2}\left\|\phi_{t}\right\|_{L^{6}}^{2} \\
& \leq \frac{\varepsilon}{2}\left\|\Delta \phi_{t}\right\|^{2}+\frac{C_{s}^{2}}{2 \varepsilon^{3}}\left\|f^{\prime}(\phi)\right\|_{L^{3}}^{2}\left\|\nabla \phi_{t}\right\|^{2}
\end{aligned}
$$

which leads to

$$
\underset{t \in[0, \infty]}{\operatorname{ess} \sup }\left\|\phi_{t}\right\|^{2}+\varepsilon \int_{0}^{\infty}\left\|\Delta \phi_{t}\right\|^{2} \mathrm{~d} t \lesssim \frac{C_{s}}{\varepsilon^{3}} \underset{t \in[0, \infty]}{\operatorname{ess} \sup }\left\|f^{\prime}(\phi)\right\|_{L^{3}}^{2} \int_{0}^{\infty}\left\|\nabla \phi_{t}\right\|^{2} \mathrm{~d} t+\left\|\phi_{t}^{0}\right\|^{2} .
$$

On the other hand side, by assumption (3.3), the Sobolev inequality (A.2) and estimate (A.9), we have

$$
\left\|f^{\prime}(\phi)\right\|_{L^{3}}^{2} \lesssim \tilde{c}_{2}\|\phi\|_{L^{3(p-2)}}^{2(p-2)}+\tilde{c}_{3} \lesssim \tilde{c}_{2}\|\phi\|_{1}^{2(p-2)}+\tilde{c}_{3} \lesssim \varepsilon^{-\left(\sigma_{1}+1\right)(p-2)}
$$

The assertion then follows from (A.14), (A.15), (ii) and assumption (3.9).

(iv) Testing (A.10) with $-\Delta^{-1} \phi_{t t}$, we get

$$
\begin{aligned}
& \left\|\phi_{t t}\right\|_{-1}^{2}+\frac{\varepsilon}{2} \frac{d}{d t}\left\|\nabla \phi_{t}\right\|^{2}=-\frac{1}{\varepsilon}\left(f^{\prime}(\phi) \phi_{t}, \phi_{t t}\right) \\
= & -\frac{1}{2 \varepsilon} \frac{d}{d t}\left(f^{\prime}(\phi) \phi_{t}, \phi_{t}\right)+\frac{1}{2 \varepsilon}\left(f^{\prime \prime}(\phi) \phi_{t}^{2}, \phi_{t}\right) \\
\leq & -\frac{1}{2 \varepsilon} \frac{d}{d t}\left(f^{\prime}(\phi) \phi_{t}, \phi_{t}\right)+\frac{1}{2 \varepsilon}\left\|f^{\prime \prime}\right\|_{L^{6}}\left\|\phi_{t}^{2}\right\|_{L^{3}}\left\|\phi_{t}\right\| \\
\leq & -\frac{1}{2 \varepsilon} \frac{d}{d t}\left(f^{\prime}(\phi) \phi_{t}, \phi_{t}\right)+\frac{C_{s}^{2}}{2 \varepsilon}\left\|f^{\prime \prime}\right\|_{L^{6}}\left\|\nabla \phi_{t}\right\|^{2}\left\|\phi_{t}\right\|
\end{aligned}
$$


Integrate (A.16) over $[0, T]$, we continue the estimate as

$$
\begin{aligned}
& 2 \int_{0}^{T}\left\|\phi_{t t}\right\|_{-1}^{2} \mathrm{~d} t+\varepsilon\left\|\nabla \phi_{t}(T)\right\|^{2}-\varepsilon\left\|\nabla \phi_{t}^{0}\right\|^{2} \\
\leq & -\left.\frac{1}{\varepsilon}\left(f^{\prime}(\phi) \phi_{t}, \phi_{t}\right)\right|_{t=T}+\frac{1}{\varepsilon}\left(f^{\prime}\left(\phi^{0}\right) \phi_{t}^{0}, \phi_{t}^{0}\right)+\frac{C_{s}^{2}}{\varepsilon} \operatorname{essup}_{t \in[0, T]}^{\operatorname{ess}}\left\{\left\|f^{\prime \prime}\right\|_{L^{6}}\left\|\phi_{t}\right\|\right\} \int_{0}^{T}\left\|\nabla \phi_{t}\right\|^{2} \mathrm{~d} t \\
\leq & \frac{\varepsilon}{2}\left\|\nabla \phi_{t}(T)\right\|^{2}+\frac{\tilde{c}_{0}^{2}}{2 \varepsilon^{3}}\left\|\phi_{t}(T)\right\|_{-1}^{2}+\frac{1}{\varepsilon}\left(f^{\prime}\left(\phi^{0}\right) \phi_{t}^{0}, \phi_{t}^{0}\right)+\frac{C_{s}^{2}}{\varepsilon} \operatorname{ess~sup}_{t \in[0, T]}\left\{\left\|f^{\prime \prime}\right\|_{L^{6}}\left\|\phi_{t}\right\|\right\} \int_{0}^{T}\left\|\nabla \phi_{t}\right\|^{2} \mathrm{~d} t,
\end{aligned}
$$
i.e.

$$
\begin{aligned}
2 \int_{0}^{T}\left\|\phi_{t t}\right\|_{-1}^{2} \mathrm{~d} t & +\frac{\varepsilon}{2}\left\|\nabla \phi_{t}(T)\right\|^{2} \leq \varepsilon\left\|\nabla \phi_{t}^{0}\right\|^{2}+\frac{1}{\varepsilon}\left(f^{\prime}\left(\phi^{0}\right) \phi_{t}^{0}, \phi_{t}^{0}\right) \\
& +\frac{\tilde{c}_{0}^{2}}{2 \varepsilon^{3}}\left\|\phi_{t}(T)\right\|_{-1}^{2}+\frac{C_{s}^{2}}{\varepsilon} \operatorname{ess~sup}_{t \in[0, T]}\left\{\left\|f^{\prime \prime}\right\|_{L^{6}}\left\|\phi_{t}\right\|\right\} \int_{0}^{T}\left\|\nabla \phi_{t}\right\|^{2} \mathrm{~d} t .
\end{aligned}
$$

On the other hand, by (3.4), the Sobolev inequality (A.2) and estimate (A.9), we have

$$
\left\|f^{\prime \prime}\right\|_{L^{6}} \lesssim \tilde{c}_{4}\|\phi\|_{L^{6(p-3)}}^{(p-3)^{+}}+\tilde{c}_{5} \lesssim\|\phi\|_{1}^{(p-3)^{+}} \lesssim \varepsilon^{-\frac{1}{2}\left(\sigma_{1}+1\right)(p-3)^{+}}
$$

By taking maximum for terms depending on $T$ in (A.18) and using (A.19), (ii), (iii) and the inequality (3.10) of Assumption 2. we obtain the assertion (iv).

(v) We formally differentiate (A.10) in time to derive

$$
\phi_{t t t}+\varepsilon \Delta^{2} \phi_{t t}=\frac{1}{\varepsilon} \Delta\left(f^{\prime \prime}(\phi)\left(\phi_{t}\right)^{2}+f^{\prime}(\phi) \phi_{t t}\right) .
$$

Testing (A.20) with $\Delta^{-2} \phi_{t t}$, we obtian

$$
\begin{aligned}
& \frac{1}{2} \frac{d}{d t}\left\|\Delta^{-1} \phi_{t t}\right\|^{2}+\varepsilon\left\|\phi_{t t}\right\|^{2}=\frac{1}{\varepsilon}\left(f^{\prime \prime}(\phi)\left(\phi_{t}\right)^{2}+f^{\prime}(\phi) \phi_{t t}, \Delta^{-1} \phi_{t t}\right) \\
\leq & \frac{\varepsilon}{2}\left\|f^{\prime \prime}(\phi)\right\|_{L^{2}}^{2}\left\|\phi_{t}\right\|_{L^{6}}^{4}+\frac{1}{2 \varepsilon^{3}}\left\|\Delta^{-1} \phi_{t t}\right\|_{L^{6}}^{2}+\frac{1}{2 \varepsilon^{3}}\left\|f^{\prime}(\phi)\right\|_{L^{3}}^{2}\left\|\Delta^{-1} \phi_{t t}\right\|_{L^{6}}^{2}+\frac{\varepsilon}{2}\left\|\phi_{t t}\right\|^{2} \\
\leq & \frac{\varepsilon}{2} C_{s}^{4}\left\|f^{\prime \prime}(\phi)\right\|_{L^{2}}^{2}\left\|\nabla \phi_{t}\right\|^{4}+\frac{C_{s}^{2}}{2 \varepsilon^{3}}\left\|\phi_{t t}\right\|_{-1}^{2}+\frac{C_{s}^{2}}{2 \varepsilon^{3}}\left\|f^{\prime}(\phi)\right\|_{L^{3}}^{2}\left\|\phi_{t t}\right\|_{-1}^{2}+\frac{\varepsilon}{2}\left\|\phi_{t t}\right\|^{2} .
\end{aligned}
$$

After taking integration from $[0, T]$ and taking maximum for terms depending on $T$, we have

$$
\begin{aligned}
& \operatorname{ess~sup}_{t \in[0, \infty]}\left\|\Delta^{-1} \phi_{t t}\right\|^{2}+\varepsilon \int_{0}^{\infty}\left\|\phi_{t t}\right\|^{2} \mathrm{~d} t \\
& \lesssim \varepsilon \operatorname{ess~sup}_{t \in[0, \infty]}\left(\left\|f^{\prime \prime}(\phi)\right\|_{L^{2}}^{2}\left\|\nabla \phi_{t}\right\|^{2}\right) \int_{0}^{\infty}\left\|\nabla \phi_{t}\right\|^{2} \mathrm{~d} t \\
& \quad+\frac{1}{\varepsilon^{3}}\left(\operatorname{ess~sup}_{t \in[0, \infty]}\left\|f^{\prime}(\phi)\right\|_{L^{3}}^{2}+1\right) \int_{0}^{\infty}\left\|\phi_{t t}\right\|_{-1}^{2} \mathrm{~d} t+\left\|\Delta^{-1} \phi_{t t}^{0}\right\|^{2} .
\end{aligned}
$$

The assertion then follows from (A.15), the following estimate

$$
\left\|f^{\prime \prime}\right\|_{L^{2}}^{2} \lesssim \tilde{c}_{4}\|\phi\|_{L^{2(p-3)^{+}}}^{(p-3)^{+}}+\tilde{c}_{5} \lesssim\|\phi\|_{1}^{2(p-3)^{+}} \lesssim \varepsilon^{-\left(\sigma_{1}+1\right)(p-3)^{+}},
$$

(ii), (iv) and the inequality (3.11) of Assumption 2

(vi) Pairing (A.20) with $-\Delta^{-3} \phi_{t t t}$, we obtain

$$
\begin{aligned}
& \left\|\Delta^{-1} \phi_{t t t}\right\|_{-1}^{2}+\frac{\varepsilon}{2} \frac{d}{d t}\left\|\phi_{t t}\right\|_{-1}^{2} \\
= & -\frac{1}{\varepsilon}\left(f^{\prime \prime}(\phi)\left(\phi_{t}\right)^{2}+f^{\prime}(\phi) \phi_{t t}, \Delta^{-2} \phi_{t t t}\right) \\
\leq & \frac{C_{s}^{2}}{\varepsilon^{2}}\left(\left\|f^{\prime \prime}(\phi)\right\|_{L^{2}}^{2}\left\|\phi_{t}\right\|_{L^{6}}^{4}+\left\|f^{\prime}(\phi)\right\|_{L^{3}}^{2}\left\|\phi_{t t}\right\|^{2}\right)+\frac{1}{2 C_{s}^{2}}\left\|\Delta^{-2} \phi_{t t t}\right\|_{L^{6}}^{2} \\
\leq & \frac{C_{s}^{2}}{\varepsilon^{2}}\left(C_{s}^{4}\left\|f^{\prime \prime}(\phi)\right\|_{L^{2}}^{2}\left\|\nabla \phi_{t}\right\|^{4}+\left\|f^{\prime}(\phi)\right\|_{L^{3}}^{2}\left\|\phi_{t t}\right\|^{2}\right)+\frac{1}{2}\left\|\Delta^{-1} \phi_{t t t}\right\|_{-1}^{2} .
\end{aligned}
$$


Integrating (A.24) from $[0, \infty)$, we have

$$
\begin{aligned}
& \int_{0}^{\infty}\left\|\Delta^{-1} \phi_{t t t}\right\|_{-1}^{2} \mathrm{~d} t+\underset{t \in[0, \infty]}{\operatorname{ess} \sup } \varepsilon\left\|\phi_{t t}\right\|_{-1}^{2} \\
\leq & \frac{2}{\varepsilon^{2}} C_{s}^{6} \operatorname{essup}_{t \in[0, \infty]}\left(\left\|f^{\prime \prime}(\phi)\right\|_{L^{2}}^{2}\left\|\nabla \phi_{t}\right\|^{2}\right) \int_{0}^{\infty}\left\|\nabla \phi_{t}\right\|^{2} \mathrm{~d} t \\
+ & \frac{2 C_{s}^{2}}{\varepsilon^{2}} \operatorname{essup}_{t \in[0, \infty]}\left\|f^{\prime}(\phi)\right\|_{L^{3}}^{2} \int_{0}^{\infty}\left\|\phi_{t t}\right\|^{2} \mathrm{~d} t+\varepsilon\left\|\phi_{t t}^{0}\right\|_{-1}^{2} .
\end{aligned}
$$

The assertion then follows from (A.23), (A.15), (ii), (iv), (v) and the inequality (3.12) of Assumption 2.

\section{References}

1. S. M. Allen and J. W. Cahn. A microscopic theory for antiphase boundary motion and its application to antiphase domain coarsening. Acta Metall. Mater., 27:1085-1095, 1979. 1

2. J. Barrett, J. Blowey, and H. Garcke. Finite element approximation of the Cahn-Hilliard equation with degenerate mobility. SIAM J. Numer. Anal., 37(1):286-318, 1999. 1

3. A. Baskaran, P. Zhou, Z. Hu, C. Wang, S. Wise, and J. Lowengrub. Energy stable and efficient finitedifference nonlinear multigrid schemes for the modified phase field crystal equation. J. Comput. Phys., 250:270-292, 2013. 1

4. B. Benesová, C. Melcher, and E. Süli. An implicit midpoint spectral approximation of nonlocal CahnHilliard equations. SIAM J. Numer. Anal., 52(3):1466-1496, 2014. 1

5. Luis A. Caffarelli and Nora E. Muler. An $L^{\infty}$ bound for solutions of the Cahn-Hilliard equation. Arch. Rational Mech. Anal., 133(2):129-144, 1995. 1, 2

6. John W. Cahn and John E. Hilliard. Free energy of a nonuniform system. I. interfacial free energy. J. Chem. Phys., 28(2):258-267, 1958. 1

7. L.Q. Chen and J. Shen. Applications of semi-implicit Fourier-spectral method to phase field equations. Comput. Phys. Commun., 108(2-3):147-158, 1998. 1

8. W. Chen, C. Wang, X. Wang, and S.M. Wise. A linear iteration algorithm for a second-order energy stable scheme for a thin film model without slope selection. J Sci. Comput., 59(3):574-601, 2014 . 1

9. Xinfu Chen. Spectrum for the Allen-Cahn, Cahn-Hillard, and phase-field equations for generic interfaces. Commun. Part. Diff. Eq., 19(7):1371-1395, 1994. 1, 3, 3

10. Nicolas Condette, Christof Melcher, and Endre Süli. Spectral approximation of pattern-forming nonlinear evolution equations with double-well potentials of quadratic growth. Math. Comp., 80(273):205-223, 2011. 1, 1, 2, 4

11. A. E. Diegel, C. Wang, and S. M. Wise. Stability and convergence of a second order mixed finite element method for the Cahn-Hilliard equation. IMA J Numer. Anal., 36(4):1867-1897, 2016. 1

12. Qiang Du and Roy A. Nicolaides. Numerical analysis of a continuum model of phase transition. SIAM J Numer. Anal., 28(5):1310-1322, 1991. 1, 1

13. C. Elliott and H. Garcke. On the Cahn-Hilliard Equation with Degenerate Mobility. SIAM J Math. Anal., 27(2):404-423, 1996. 1

14. C. M. Elliott and A. M. Stuart. The global dynamics of discrete semilinear parabolic equations. SIAM J. Numer. Anal., 30:1622-1663, 1993. 1

15. Charles M. Elliott and Stig Larsson. Error estimates with smooth and nonsmooth data for a finite element method for the Cahn-Hilliard equation. Math. Comp., 58(198):603-630, S33-S36, 1992.1

16. D. J. Eyre. Unconditionally gradient stable time marching the Cahn-Hilliard equation. In Computational and Mathematical Models of Microstructural Evolution (San Francisco, CA, 1998), volume 529 of Mater. Res. Soc. Sympos. Proc., pages 39-46. MRS, 1998. 1, 1

17. X. Feng. Fully discrete finite element approximations of the Navier-Stokes-Cahn-Hilliard diffuse interface model for two-phase fluid flows. SIAM J. Numer. Anal., 44(3):1049-1072, 2006. 1

18. Xiaobing Feng and Andreas Prohl. Error analysis of a mixed finite element method for the Cahn-Hilliard equation. Numer. Math., 99(1):47-84, 2004. 1, 1, 4

19. Xiaobing Feng and Andreas Prohl. Numerical analysis of the Cahn-Hilliard equation and approximation for the Hele-Shaw problem. Interfaces Free Bound., 7(1):1-28, 2005. 2

20. Xinlong Feng, Tao Tang, and Jiang Yang. Stabilized Crank-Nicolson/Adams-Bashforth schemes for phase field models. E Asian J Appl. Math., 3(1):59-80, 2013. 1 
21. Daisuke Furihata. A stable and conservative finite difference scheme for the Cahn-Hlliard equation. Numer. Math., 87(4):675-699, 2001. 1, 1

22. Hector Gomez and Thomas J. R. Hughes. Provably unconditionally stable, second-order time-accurate, mixed variational methods for phase-field models. J. Comput. Phys., 230(13):5310-5327, 2011. 1

23. F. Guilln-Gonzlez and G. Tierra. On linear schemes for a Cahn-Hilliard diffuse interface model. $J$. Comput. Phys., 234:140-171, 2013. 1

24. Francisco Guilln-Gonzlez and Giordano Tierra. Second order schemes and time-step adaptivity for Allen-Cahn and Cahn-Hilliard models. Comput. Math. Appl., 68(8):821-846, 2014. 1

25. Jing Guo, Cheng Wang, Steven M. Wise, and Xingye Yue. An $H^{2}$ convergence of a second-order convexsplitting, finite difference scheme for the three-dimensional Cahn-Hilliard equation. Commun. Math. Sci, 14(2):489-515, 2016. 1

26. Ruihan Guo, Francis Filbet, and Yan Xu. Efficient high order semi-implicit time discretization and local discontinuous Galerkin methods for highly nonlinear PDEs. J Sci Comput, 68(3):1029-1054, 2016. 1

27. D. Han, A. Brylev, X. Yang, and Z. Tan. Numerical analysis of second order, fully discrete energy stable schemes for phase field models of two phase incompressible flows. J. Sci. Comput., 70:965-989, 2017. 1

28. Yinnian He, Yunxian Liu, and Tao Tang. On large time-stepping methods for the Cahn-Hilliard equation. Appl. Numer. Math., 57(5-7):616-628, 2007. 1

29. Lili Ju, Jian Zhang, and Qiang Du. Fast and accurate algorithms for simulating coarsening dynamics of Cahn-Hilliard equations. Computational Materials Science, 108, Part B, 2015. 1

30. Daniel Kessler, Ricardo H. Nochetto, and Alfred Schmidt. A posteriori error control for the Allen-Cahn problem: circumventing Gronwall's inequality. ESAIM: Math. Model. Numer. Anal., 38(01):129-142, 2004. 1, 2, 4

31. Junseok Kim, Kyungkeun Kang, and John Lowengrub. Conservative multigrid methods for CahnHilliard fluids. J. Comput. Phys., 193(2):511-543, 2004. 1

32. Dong Li and Zhonghua Qiao. On second order semi-implicit Fourier spectral methods for 2d CahnHilliard equations. J Sci. Comput., 70(1):301-341, 2017. 1

33. Dong Li, Zhonghua Qiao, and Tao Tang. Characterizing the stabilization size for semi-implicit Fourierspectral method to phase field equations. SIAM J Numer. Anal., 54(3):1653-1681, 2016. 1

34. Chun Liu and Jie Shen. A phase field model for the mixture of two incompressible fluids and its approximation by a Fourier-spectral method. Physica D, 179(3-4):211-228, 2003.

35. F Magaletti, Francesco Picano, M Chinappi, Luca Marino, and Carlo Massimo Casciola. The sharpinterface limit of the Cahn-Hilliard/Navier-Stokes model for binary fluids. J Fluid. Mech., 714:95-126, 2013. 2

36. J. Shen, J. Xu, and J. Yang. The scalar auxiliary variable (SAV) approach for gradient flows. J. Comput. Phys., 353:407-416, 2017. 1

37. Jie Shen, Jie Xu, and Jiang Yang. A new class of efficient and robust energy stable schemes for gradient flows. arXiv:1710.01331, 2017. 1

38. Jie Shen and Xiaofeng Yang. Numerical approximations of Allen-Cahn and Cahn-Hilliard equations. Discrete Cont. Dyn. A, 28:1669-1691, 2010. 1, 1, 2, 4

39. Jie Shen, Xiaofeng Yang, and Haijun Yu. Efficient energy stable numerical schemes for a phase field moving contact line model. J. Comput. Phys., 284:617-630, 2015. 4.1

40. Jaemin Shin, Hyun Geun Lee, and June-Yub Lee. Unconditionally stable methods for gradient flow using Convex Splitting RungeKutta scheme. Journal of Computational Physics, 347:367-381, 2017. 1

41. Lin Wang and Haijun Yu. Convergence analysis of an unconditionally energy stable linear CrankNicolson scheme for the Cahn-Hilliard equation. J. Math. Study, 51(1):89-114, 2017. 3

42. Lin Wang and Haijun Yu. Energy stable second order linear schemes for the Allen-Cahn phase-field equation. Commun. Math. Sci., in revision, 2018. 1

43. X. Wu, G. J. van Zwieten, and K. G. van der Zee. Stabilized second-order convex splitting schemes for Cahn-Hilliard models with application to diffuse-interface tumor-growth models. Int. J. Numer. Meth. Biomed. Engng., 30(2):180-203, 2014. 1

44. C. Xu and T. Tang. Stability analysis of large time-stepping methods for epitaxial growth models. SIAM J. Num. Anal., 44:1759-1779, 2006. 1

45. Xianmin Xu, Yana Di, and Haijun Yu. Sharp-interface limits of a phase-field model with a generalized Navier slip boundary condition for moving contact lines. J. Fluid Mech., to appear(arXiv:1710.09141), 2018. 2

46. Xiaofeng Yang. Linear, first and second-order, unconditionally energy stable numerical schemes for the phase field model of homopolymer blends. J. Comput. Phys., 327:294-316, 2016. 1

47. Xiaofeng Yang and Lili Ju. Efficient linear schemes with unconditional energy stability for the phase field elastic bending energy model. Comput. Method. Appl. Mech. Eng., 315:691-712, 2017. 1

48. Xiaofeng Yang and Haijun Yu. Efficient second order unconditionally stable schemes for a phase field moving contact line model using an invariant energy quadratization approach. SIAM J. Sci. Comput., to appear, 2018. 1 
49. Haijun $\mathrm{Yu}$ and Xiaofeng Yang. Numerical approximations for a phase-field moving contact line model with variable densities and viscosities. J. Comput. Phys., 334:665-686, 2017. 4.1

50. Zhengru Zhang, Yuan Ma, and Zhonghua Qiao. An adaptive time-stepping strategy for solving the phase field crystal model. J. Comput. Phys., 249:204-215, 2013. 1

51. Jingzhi Zhu, Long-Qing Chen, Jie Shen, and Veena Tikare. Coarsening kinetics from a variablemobility Cahn-Hilliard equation: Application of a semi-implicit Fourier spectral method. Phys. Rev. E, 60(4):3564-3572, 1999. 